See discussions, stats, and author profiles for this publication at: https://www.researchgate.net/publication/312133761

\title{
Erosion prediction of industrial conveying pipelines
}

Article in Powder Technology · March 2017

DOI: 10.1016/j.powtec.2016.12.087

CITATIONS

21

3 authors:

C.) Avi Uzi

Ben-Gurion University of the Negev

19 PUBLICATIONS 118 CITATIONS

SEE PROFILE

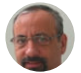

Avi Levy

Ben-Gurion University of the Negev

197 PUBLICATIONS 3,286 CITATIONS

SEE PROFILE

Some of the authors of this publication are also working on these related projects:

Project Energy absorption in particle-wall impact View project

Project Erosion and particle breakage in conveying systems View project
READS

311

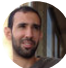

Yaron Ben Ami

Technion - Israel Institute of Technology

11 PUBLICATIONS 77 CITATIONS

SEE PROFILE 


\section{Erosion Prediction of Industrial Conveying Pipelines}

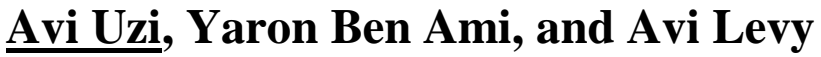

Department of Mechanical Engineering, Ben-Gurion University of the Negev, Beer Sheva 8410501, Israel

ABSTRACT

Obtaining the particles' collision characteristics from 3D simulations
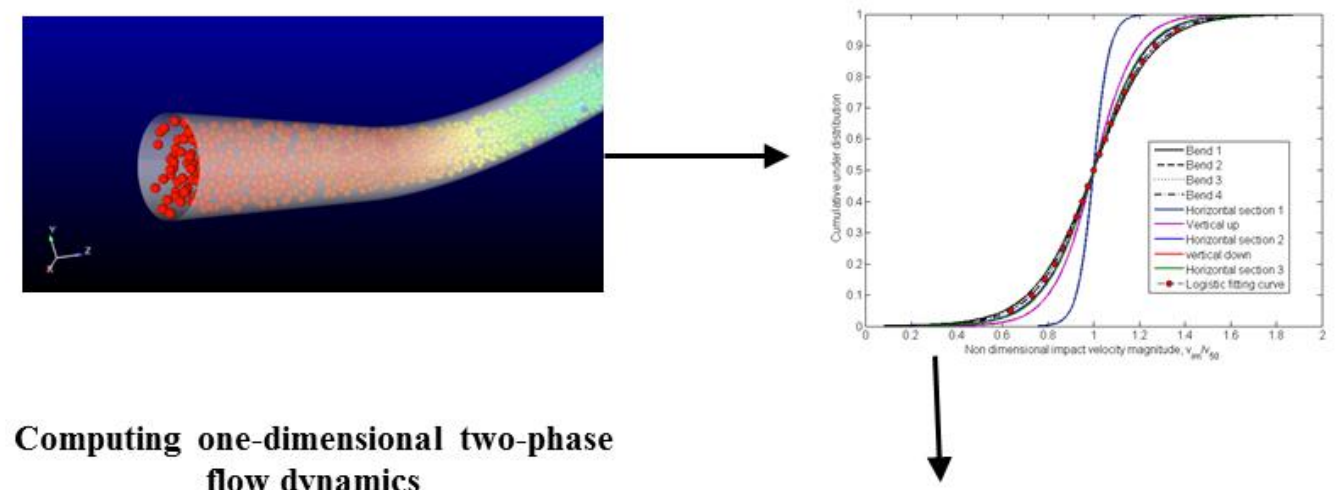

Computing one-dimensional two-phase flow dynamics

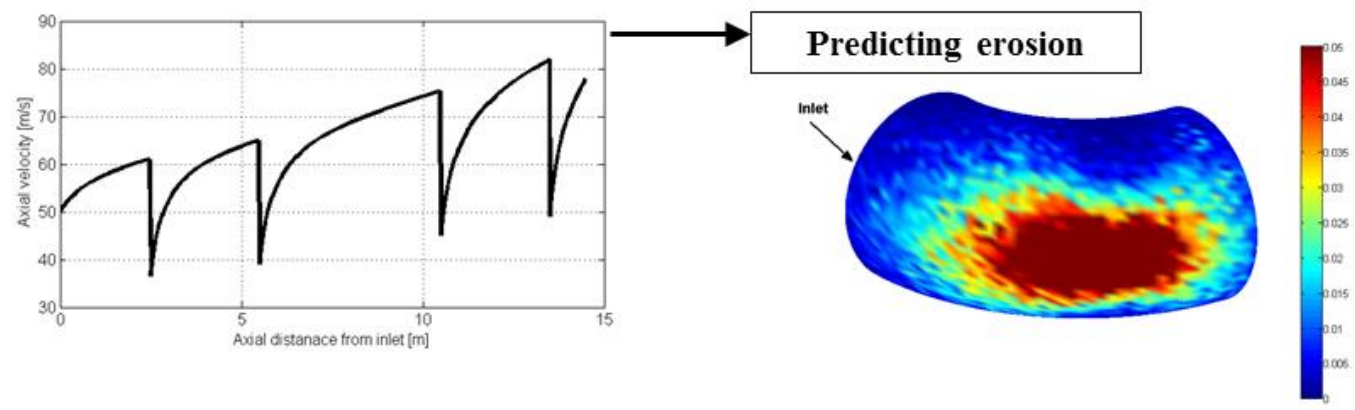

In this paper, a new concept of the one-dimensional erosion model (ODEM) is presented, validated, and implemented for a pneumatic conveying system. The ODEM is a combination of a one-dimensional flow model and statistical distribution functions that describe the particle-wall collision characteristics. Computational fluid dynamics/discrete element method (CFD-DEM) simulations serve as a numerical tool for deriving the statistical functions of the collision characteristics and as a basis for ODEM validation. Both the ODEM and the CFD-DEM are implemented for the same pneumatic conveying system in order to compare their performances. The developed statistical functions for straight pipes and bends include collision frequency, circumference angle, impact angle, and impact velocity magnitude. An additional bend angle function is developed for bends. It is found that most of the parameters can be 
formulated by a single distribution function that describes all the sections of the system. A distinct feature is discovered, i.e., using a non-dimensional form of the impact velocity function (the ratio of the impact velocity magnitude to the median impact velocity) yields similar statistical distributions for all bends and straight sections in the conveying system. Furthermore, the median impact velocity is correlated with particle axial velocity. Therefore, this non-dimensional form can be used in a wide range of applications for estimating the distribution of the impact velocity of particles in dilute pneumatic conveying. The statistical functions describe each parameter independently, which means that they can be measured or computed regardless of one another, thereby facilitating fast and easy erosion prediction. The erosion rate results of the ODEM and the DEM for the entire conveying system are found to be in excellent agreement (straight sections and bends). This validates the ODEM as a tool for predicting the erosion rate in conveying lines, including bends and straight sections, at a much faster rate than 3D CFD-DEM simulations (the computation time ratio is estimated to be $1 / 54$ ).

KEYWORDS: Erosive Wear; Erosion Model; One-Dimensional Erosion Model; ODEM; CFD-DEM simulations; Pneumatic Conveying.

\section{Introduction}

Erosive wear of pipe components owing to the conveyance of solid particles is a major industrial problem. The collisions between the conveyed particles and the walls cause material removal and thus necessitate regular, expensive maintenance operations. Evaluation of the erosion rate along the conveying line and identification of the critical failure points could lead to efficient maintenance and minimization of unnecessary expenses.

In recent years, the following has been the most widely used approach for erosion prediction of piping components. First, computational fluid dynamics/discrete element method (CFD-DEM) simulation is performed to obtain the dynamic properties (i.e., impact velocity, angle, and frequency) of particle-wall impacts at each discrete location along the piping component wall. Then, the single-particle-impact erosion model (e.g., Oka et al. [1], Ahlert [2], Zhang et al. [3]) is applied to the collision data and summation is performed to obtain the discrete wear depth along the pipe component.

This approach has been adopted in many studies (Solnordal et al. [4], Vieira et al. [5], Peng \& Cao [6] are some recent examples), and it can yield good agreement with experimental data. However, it is limited by the high computational requirements of the CFD-DEM simulation, which restricts it to erosion prediction of a single pipe component (usually, bends) rather than the entire industrial conveying system. Thus, there is a need to develop a model that can overcome these limitations. Zhang et al. [7] proposed a probability model for one-dimensional erosion in long, straight pipes, where the impact dynamics is a function of the 
random radial fluctuating velocity of the turbulent flow. Liu et al. [8] proposed a probability model for three-dimensional bend erosion; they assumed two particle impacts with the bend wall, where the first impact depends only on the bend geometry and the second impact depends on the location of the first impact (which defines the return angle) together with the stochastic rebound model of Tabakoff et al. [9]. While the models of Zhang et al. [7] and Liu et al. [8] are rather simple and easy to obtain for realistic conveying systems, they do not consider the effects of particle-particle collisions, particle-wall friction, mean flow drag on the particles, particle rotation, gravity, and particle inertia.

\section{Present Study}

The main objective of the current study is to provide a useful tool for predicting the erosion rate in conveying lines with low computational requirements while providing high accuracy. Recently, a new model has been developed in a related research field, i.e., a model for attrition prediction of particles in pneumatic conveying lines [10]. This model has two components: a one-dimensional two-phase flow model that calculates the flow dynamics and a one-dimensional breakage algorithm that accounts for particle breakage. In this model, particle breakage is accounted for by combining two tools: a tool that describes the collisions dynamics, i.e., impact frequency and velocity, and empirical comminution functions that characterize the particle response to a collision. The collisions dynamics is obtained by $3 \mathrm{D}$ CFD-DEM simulation and the data are recorded for subsequent analysis. From these data, the statistical distribution functions of the collision velocity and frequency are developed.

A similar approach is adopted herein with extensive modifications required for erosion prediction. Instead of breakage calculations, the single-particle-impact erosion model is used [11], and the collision dynamics tool is expanded to predict not only the impact velocity and frequency but also the 3D locations of the particle-wall impacts in straight pipes and bends.

When modeling erosive wear in conveying lines, the following requirements must be satisfied. First, a CFD model should predict the particle dynamics along the conveying line, and every impact with the pipe wall in the 3D domain must be accounted for. Second, the single-particle-impact erosion model uses the collision data to predict the amount of the material removal and the wear distribution. Therefore, the new one-dimensional erosion model (ODEM) combines two tools: a one-dimensional two-phase flow model together with statistical functions that describe the particle-wall impact characteristics. The onedimensional two-phase model solves the steady-state balance equations of mass, momentum, and energy (discussed in detail in [10]). This tool facilitates the prediction of particle axial velocity, volume fraction, and piping pressure drop in the conveying system. The particle-wall impact characteristics are obtained by exploiting statistical functions. These functions describe the collision velocity and frequency (following the same principles specified in [10]) as well as the impact angle, circumference pipe impact 
location, and bend angle. All the above-mentioned functions are developed in the present study by fitting the collision data collected during the 3D CFD-DEM simulations to the statistical distribution functions. To bound the model, the single-particle-impact model of Ben Ami et al. [11] is adopted to define the erosion rate at a specific point, where the input parameters are impact velocity and angle, particle diameter, and empirical material constant, which account for both the target and the abrasive materials.

Once a statistical distribution function is correlated for a specific case (particle properties, bend radius, inlet velocity, etc.), these data are saved for further analysis. By performing additional CFD-DEM simulations under different conditions, the statistical distribution functions can be extended to account for a range of conditions. In contrast, using the direct method necessitates a specific CFD-DEM simulation for every change in the particles, fluid, or geometric conditions. The statistical approach is an ongoing effort, where the data collected at each simulation are added to the existing data in order to extend the range of applicability of the probability functions. With the probability functions at hand, the ODEM can simulate any desired case to predict the erosion rate along the pipeline with relatively low computational requirements. However, it should be noted that the maximum erosion prediction accuracy achieved using the ODEM is equal to that achieved using direct 3D CFD-DEM simulations because the probability functions are derived from the 3D CFD-DEM simulations.

\section{One-dimensional Erosion Model (ODEM)}

For dilute phase conveying systems, when the particle phase is fully suspended, the particles can be modeled by a homogenous suspension. Therefore, the dynamics of the particles can be evaluated by applying a one-dimensional flow model [10]. This model solves the steady-state balance equations of mass, momentum, and energy in order to predict the pressure drop, particle axial velocity, and particle volume fraction. However, it is unable to describe the particle-wall impact characteristics.

For this reason, the particle-wall impact characteristics are obtained by exploiting statistical distribution functions. This concept is adopted from the collision search algorithm of Uzi et al. [10]; in this work, it is implemented without accounting for particle breakage, while some other modifications are carried out and detailed. The original algorithm extracts the collision characteristics through 3D CFD-DEM simulations and subsequently analyzes the simulation data to provide statistical distribution functions of the collision frequency and velocity, with respect to the system's governing parameters (i.e., particle size, pipe geometry, gas superficial velocity, particle mass flux, etc.).

In erosion modeling, in addition to the velocity and frequency of collisions, the 3D locations of the collisions and their impact angles must be accounted for. However, when modeling the particles phase as a homogenous suspension with a one-dimensional model, only the axial locations of the collisions along 
the pipeline are known. Therefore, in order to obtain the impact circumference locations on the pipe wall, another statistical distribution function should be developed.

Unlike the dynamics inside straight pipe sections, the complicated dynamics inside bends cannot be predicted by the one-dimensional model. Therefore, the ODEM introduces additional statistical distribution functions to describe the bend angle impact location and circumference location inside bends.

In the two-phase flow model, the pipeline geometry is divided into equally sized numerical cells, and the model is then solved in order to obtain the steady-state two-phase flow dynamics. From this solution, the mass of the solid phase in each numerical cell along the pipeline is known. Therefore, the ODEM is initialized with a known particle size distribution at the inlet and follows it by choosing a "tracking mass". The tracking mass is a constant scalar that represents a certain amount of discrete particles. For such particles, the collision time and place are calculated along the conveying line. The collision search algorithm of Uzi et al. [10] is briefly outlined as follows for the sake of completeness.

Every discrete particle in the tracking mass has the following properties: id, diameter, and next collision time. The sum of all discrete particle masses gives the tracking mass:

$$
m_{n}=\frac{\pi}{6} \rho_{p} \sum_{i=1}^{n_{0 t}} d_{i}^{3}
$$

where $n_{t o t}$ is the number of particles in the particular element under consideration. These discrete particles fit the defined inlet particle size distribution. In order to account for the erosion along the pipe, the collisions during solid particle transport are determined using the frequency function to obtain every particle's next collision time within the domain. On the other hand, the time spent by the tracking mass inside every element is known from the flow model because the velocity of the particles phase is calculated.

Equivalently to the discrete representation of the particles in the tracking mass, the tracking mass within the continuous flow model domain is given by

$$
m_{n}=\varepsilon_{p} A \rho_{p} D X
$$

where $\varepsilon_{p}$ is the particle phase volume fraction, $A$ is the pipe cross section area, $\rho_{p}$ is the particle density, and $D X$ is the element size of the collision search algorithm. Note that from this point onward, the term "element" refers to the collision search algorithm while the term "cell" refers to the flow model.

Since the tracked mass is constant and the particle volume fraction is varied along the conveying line, the element size of the collision search algorithm must change accordingly along the pipeline to satisfy Eq. 
(2). Therefore, the coupling between the collision search algorithm and the two-phase flow model is achieved as follows. The next element size is calculated in terms of the particle volume fraction in the previous element:

$$
D X_{n e x t}=\frac{m_{n}}{\varepsilon_{p, p r e v} A \rho_{p}}
$$

It should be emphasized that we considered the previous element size at the right hand of eq. (3) to satisfy the next element size. This can be done if the volume fraction gradients along the pipeline are small. Nevertheless, iterative procedure should be considered if one would like to obtain the local element size with better accuracy on the account of computational costs.

The residence time of the particle within the cells is calculated in the flow model by

$$
\frac{d t}{d x}=\frac{1}{u_{p}}
$$

where $u_{p}$ is the particle phase velocity, $t$ denotes time, and $d x$ is the flow cell size. Thus, the residence time within each element (the time interval of the tracked mass in the element) is known and can be used in the collision search algorithm. Every tracked particle is checked to determine whether it collides with the walls, while the collision time is obtained by exploiting the frequency distribution function. If a collision is found, the impact velocity magnitude, collision angle, circumference angle, and bend angle (only in bends) are calculated from the statistical distribution functions.

The proposed ODEM is examined and validated in two steps. The first step is to develop a reliable 3D simulation that can accurately predict the particle dynamics and therefore ensure that our statistical distribution functions will be developed on the basis of a valid simulation. In the current study, CFDDEM coupling is chosen as the tool for deriving the collision characteristic functions. When using CFDDEM, many sub-models are adopted with constants that are suitable for the particles and the pipe wall properties. However, many uncertainties remain regarding the combination of all the applied models. For this reason, our CFD-DEM is tested by comparing the calculated erosion rate results obtained using the discrete erosion model of Ben Ami et al. [11] with experimental data. A valid comparison would imply that the particle dynamics is predicted accurately. Our current DEM version is modified by integrating new models that are found to be adequate for the prediction of the erosion rate with respect to the experimental results. The CFD-DEM is examined for a short bend section $(\mathrm{R} / \mathrm{D}=1.5)$ and the results are compared with the experimental erosion data of Solnordal et al. [4]. Through this stage, we confirm that the combination of all models within the CFD-DEM is valid; therefore, this tool is suitable for obtaining the collision characteristic functions. 
The second step is to use the ODEM to predict the erosion rate in a conveying line. Using the same CFDDEM setup as that used for the validation, a dilute phase pneumatic conveying system consisting of five straight sections and four bends, with a total length of $\sim 15 \mathrm{~m}$, is simulated. The simulation results are used to obtain the erosion rate directly from the raw CFD-DEM data at every point along the pipe wall. On the other hand, the CFD-DEM collision data are subsequently analyzed to provide the statistical distribution functions that describe the collision characteristics. These functions are implemented in the ODEM, and then, the same system and boundary conditions as those in the CFD-DEM simulation are simulated using the ODEM. In order to show that the ODEM statistical approach provides good assessments, both the direct 3D CFD-DEM and the ODEM erosion results are compared.

\section{CFD-DEM Establishment and Examination}

Many erosion models, analytical and empirical, have been developed over the years ( [1-3] , [12-15] ), since the first model of Finnie [16]. The recently developed single-particle-impact erosion model of BenAmi et al. [11] is a semi-analytical erosion model that captures the trends of impingement angle and velocity effects, while using three empirical coefficients that are related to target material and abrasive particles properties. This model yields good agreement with experimental data, especially in predicting the maximum erosion impingement angle, which is crucial in erosion predictions. The model gives the following erosion equation for material removal per particle mass:

$$
\frac{\Delta Q}{m_{p}}=D \cdot\left(U_{0} \sin \alpha\right)^{2.8}+C \cdot\left(1-\exp \left(-200 \alpha^{2}\right)\right) d_{p}^{1-f} \cdot U_{0}^{3-f} \cos ^{2}(\alpha) \sin ^{1-f}(\alpha)
$$

where $\Delta Q$ is the volume of material removal; $m_{p}$ and $d_{p}$ are the particle mass and diameter, respectively; $U_{0}$ is the particle impact velocity; $\alpha$ is the impact angle; and $C, D, f$ are the empirical coefficients that account for the target material and particle properties.

The model coefficients were correlated using the experimental erosion data obtained by Wong et al. for an Aluminum 6061 cylinder with sand particles [17]. The experimental erosion data and the fitted erosion equation are presented in Fig. 1, where a good fit is shown for a range of impact angles and velocities. The model coefficients obtained by the fitting are $f=0.6, D=1.374 \times 10^{-4}, C=3.641 \times 10^{-3}$. 


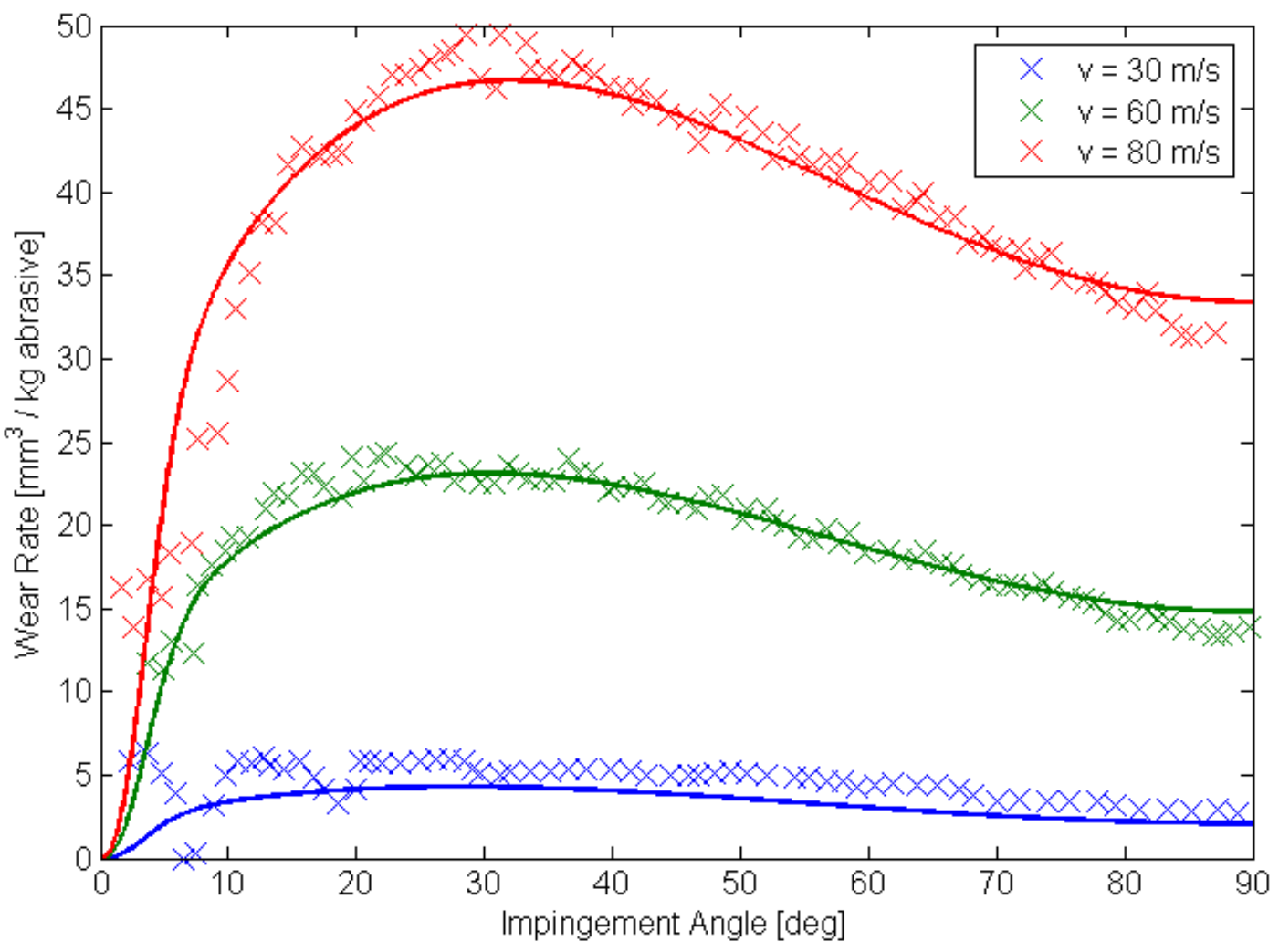

Fig. 1. Fitting the erosion equation, i.e., Eq. (5) (represented by lines), to the experimental erosion data (denoted by $X$ in the figure) of Wong et al. [17].

\subsection{CFD-DEM Simulation Setup}

The computational domain was calculated for 3D two-phase flow using ANSYS-FLUENT 16.2 as the gas phase solver and our in-house BGU-DEM ${ }^{1}$ model [18],[19] as the solver for the discrete particle motion. Pressure based solver with compressible air as ideal gas was assumed and the boundary conditions were set to velocity-inlet and pressure-outlet. The particle mass flux was set to $0.03 \mathrm{~kg} / \mathrm{s}$ and the air mass flux was $0.78 \mathrm{~kg} / \mathrm{s}$. The inlet velocity conditions of both the fluid and the particles were determined by conducting CFD-DPM (discrete phase model; ANSYS-FLUENT 16.2) simulation of the entire experimental system of Solnordal et al. [4]. The CFD-DPM simulation was performed for the entire conveying system in order to evaluate the right inlet conditions for the DEM simulation; in terms of the computational time, it is not feasible to simulate the entire system using the DEM. The DPM simulation yielded the velocity distribution of the particles and the fluid in the pipe inlet section of the DEM simulation. These values were integrated into the DEM simulation by a user-defined function. It is worth noting that owing to the existence of previous bends in the conveying system, the velocity at the DEM

\footnotetext{
${ }^{1}$ The BGU-DEM model is an in-house discrete particle model that was developed at the Department of Mechanical Engineering, Ben-Gurion University of the Negev. For further details, please refer to [18],[19].
} 
inlet section had both radial and tangential components that affected the erosion results. The DEM setup conditions for the simulated sand particles are summarized in Error! Reference source not found..

Table 1 Particle and wall properties in DEM simulations

\section{Particle properties}

Poisson's ratio

Young's modulus

Density

Coefficient of restitution (particle-wall)

Coefficient of restitution (particle-particle)

Diameter
0.2

$70 \mathrm{GPa}$

$2650 \mathrm{~kg} / \mathrm{m}^{3}$

0.75

0.9

80-350 $\mu \mathrm{m}$

The forces exerted on the particles include drag, gravity, buoyancy, rotational lift, shear lift, wall collisions, and particle collisions. These forces have been fully detailed in previous studies [18],[19]. The drag coefficient is calculated using the model of Haider \& Levenspiel [20], which takes the particle sphericity into account. The sphericity used was 0.7 , a typical sphericity of sand particles [21]. In addition, the random walk model of Gosman and Ioannides [22] was adopted and integrated into the DEM code. This modifies the drag force according to the calculated stochastic drag force in terms of the local turbulent parameters $k$ and $\varepsilon$. Since only dilute phase pneumatic conveying is considered in this paper, one-way coupling (i.e., where the particle dynamics does not affect the fluid dynamics) was implemented in all our CFD-DEM simulations.

\subsubsection{Particle-Wall Rebound}

Owing to wall roughness and irregularity of the sand particle shape, the DEM rebound model of spherical particles with a smooth wall (Tsuji [23]) leads to inaccurate rebound of the sand particles when they collide with a rough wall. In practice, the ductile surface is far from being smooth, as was clearly shown by Cenna et al. [24] that the eroded surface is cracked and lips like shapes and ripples are formed, all due to repeated particle impacts.

The particle rebound properties significantly influence the extent of erosion in bends [4] owing to the importance of the second and third impacts events, which are affected by the rebound characteristics. In order to deal with this problem, Sommerfeld and Huber [25] developed the stochastic rebound model. This model assumes that the rebound angle consists of the impact angle and the supplement of the stochastic normally distributed angle:

$$
\alpha_{r}=\alpha_{i}+\Delta \gamma \xi
$$


where $\alpha_{r}$ is the rebound angle, $\alpha_{i}$ is the impact angle, $\xi$ is a normally distributed random number with zero mean and unity standard deviation, and $\Delta \gamma$ is the stochastic angle standard deviation that depends on the particle shape and wall roughness. The value of $\Delta \gamma$ is difficult to evaluate and needs to be calibrated through direct impact experiments. In addition, this model allows a change in the rebound angle only in the plane defined by the wall normal and the velocity direction. Note that in realistic cases, a rough wall can produce a rebound angle component in the perpendicular plane. However, during the erosion process, the shape of the wall surface can be assumed to consist of dimples created by particle impacts, and these dimples in turn are eliminated and a new dimple is formed. Thus, if we can obtain a valid estimation of the size of the dimple, we can evaluate the rebound angle. Under this assumption, we developed a dimple rebound model that calculates a stochastic rebound angle in terms of the characteristic dimple properties.

\subsubsection{Dimple Rebound Model (DRM)}

Consider the following situation, which is presented in Fig. 2. A surface with a normal $\hat{n}_{w}$ that is covered by spherical cap dimples of known base radius $R_{c}$ and depth $y$ is impacted by a particle with impact angle $\alpha_{i}$. By determining the location where the particle tip intersects with the dimple base, defined by $a \hat{k}+b \hat{t}$ , we can evaluate the normal at the impact location $\hat{n}_{d}$, and then, the rebound angle $\alpha_{r}$ is determined. The location of intersection is random because we only assume that the wall surface is fully covered with dimples. However, there is uncertainty regarding the exact location of each dimple. Hence, we can assume that $a, b$ can be calculated as follows:

$$
\begin{aligned}
& a=r_{r} \cdot \cos \left(\psi_{r}\right) \\
& b=r_{r} \cdot \sin \left(\psi_{r}\right)
\end{aligned}
$$

where $r_{r}$ is a uniformly random number in the range $\left[0, R_{c}\right]$ that describes the distance of the random point from the dimple base center, $R_{c}$ is a the dimple base radius that is considered constant, and $\psi_{r}$ is a uniformly random angle in the range $[0,2 \pi]$ that describes the circumferential location on the dimple base. Therefore, this representation allows covering statistically different intersection locations between the dimple surface and the particle tip while considering constant dimple morphology. 

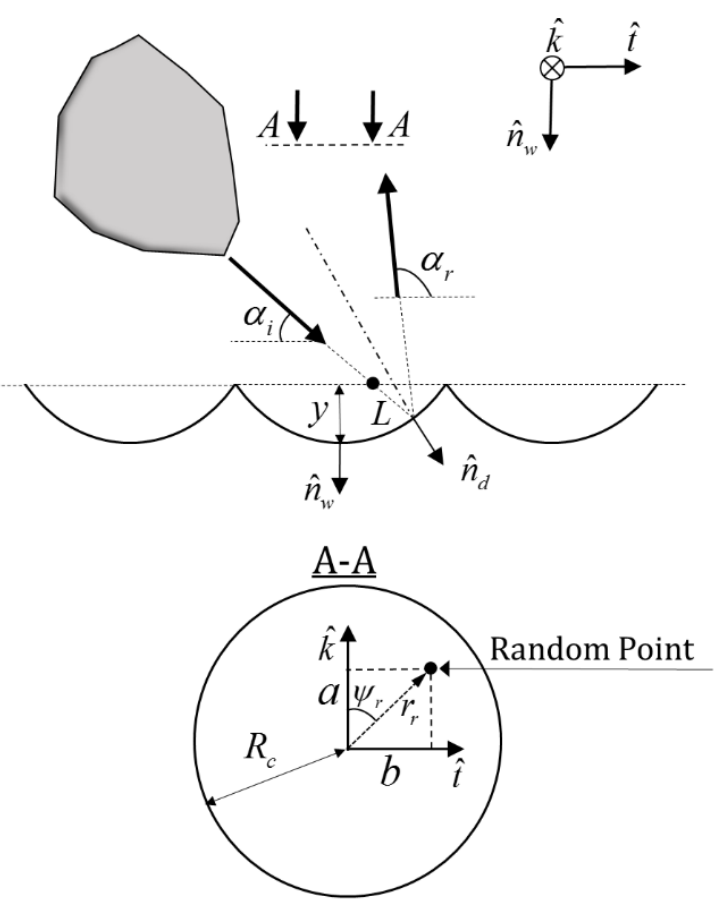

Fig. 2. Schematic illustration of DRM geometric parameters.

The new normal $\hat{n}_{d}$ is calculated as follows:

$$
\hat{n}_{d}=\cos \vartheta \hat{n}_{w}+\sin \vartheta \sin \varphi \hat{t}+\sin \vartheta \cos \varphi \hat{k}
$$

where $\varphi$ is the azimuthal angle (the angle between the projection of $\hat{n}_{d}$ on the plane $\hat{k}-\hat{t}$ and the $\hat{k}$ axis) and $\vartheta$ is the polar angle (the angle between $\hat{n}_{d}$ and the $\hat{n}_{w}$ axis). These angles, which describe the location of impact on the dimple wall, are calculated as

$$
\begin{gathered}
\tan \varphi=\frac{b+L \cos \alpha}{a} \\
\cos \vartheta=\frac{R_{s}+L \sin \alpha-y}{R_{s}}
\end{gathered}
$$

where $L$ is the distance between a random crossing location and the dimple wall:

$$
L=\left(y-R_{s}\right) \sin \alpha-b \cos \alpha+\sqrt{\left[\left(y-R_{s}\right) \sin \alpha-b \cos \alpha\right]^{2}-\left[y^{2}+a^{2}+b^{2}-2 y R_{s}\right]}
$$

$R_{s}$ is the radius of the sphere, which is related to the cap depth as 


$$
y=R_{s}\left(1-\sqrt{1-\beta^{2}}\right)
$$

where $\beta$ is the ratio of the cap base radius to the sphere radius: $\beta=R_{c} / R_{s}$. This relation is a nondimentional proerty that can help defining the cap size while the sphere size is known. The sphere radius $R_{s}$ in our simulations was naturally chosen to be at the size of the average particle: $107 \mu \mathrm{m}$. But to complete the DRM model $\beta$ must be evaluated.

For this reason the proper ratio of $\beta$ was estimated using the experimental data of Oka et al. [26]. In his experiments Oka measured $\beta$ at indentations produced by sand particles having a diameter of $215 \mu \mathrm{m}$ at a normal impact of $100 \mathrm{~m} / \mathrm{s}$ for various target materials. From these measurement Oka correlated $\beta$ with the material hardness: $\beta=k(H V)^{-n}$. Therefore, from this correlation we can obtain $\beta$ if the material hardness is known.

The hardness of the pipe wall in our simulations is HV $=56.4$ (Aluminum 6061, Wong et al. [17]), and its corresponding value of $\beta$ is 0.34 . Therefore, for the particle radii of $107 \mu \mathrm{m}$, the cap base radius is 34.4 $\mu \mathrm{m}$.

\subsection{Validation Results}

The wear depth obtained in the simulation in four different paths on the bend wall (circumference angles of $0^{\circ}, 20^{\circ}, 40^{\circ}$, and $60^{\circ}$, where $0^{\circ}$ accounts for the path with the largest curvature radius) was validated against the experimental depth (see Fig. 3). The contribution of the developed DRM to the accuracy of the erosion prediction is shown in Fig. 3 and Fig. 4. When the DRM is not implemented, as shown in Fig. 3A and Fig. 4A, the cylindrical pipe wall tends to concentrate the impact of secondary particles on the center line $\left(\phi=0^{\circ}\right)$. This impact concentration creates a high peak of erosion on the center line and a "V"-shaped erosion scar in the area after the peak site (as can be seen in Fig. 4A). This erosion scar is not observed in the experimental data and is a deficiency of the smooth wall impact model, which is commonly used in DEM simulations. When using the DRM, the results show very good agreement for the $0^{\circ}$ path (designated in Fig. 3B), where the erosion is maximized, with very little error at the maximum erosion location. In addition, the locations of maximum erosion in each path were predicted correctly, suggesting that the dynamics of the particles in the bend was calculated correctly in the CFD-DEM simulation. 
A
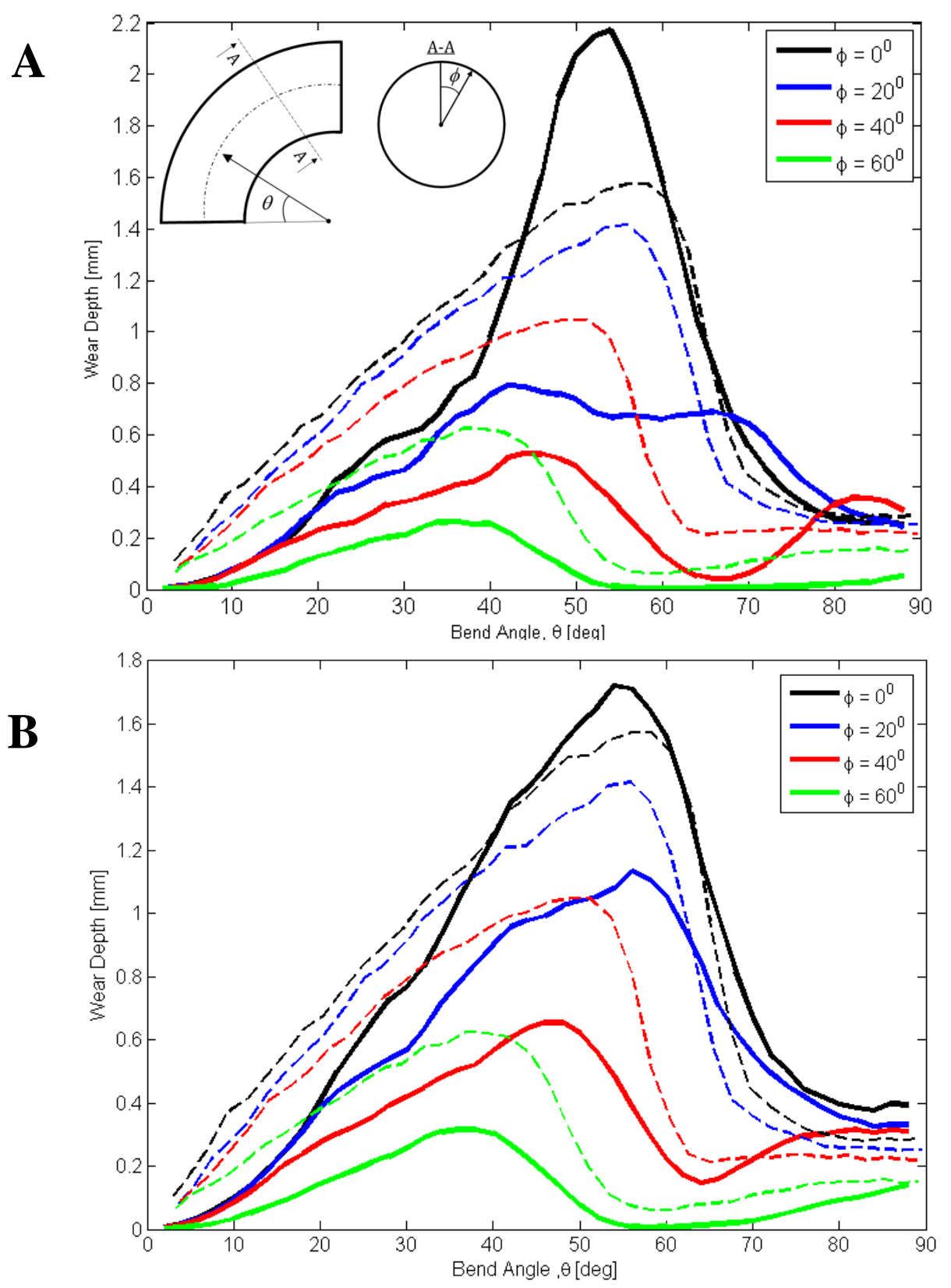

Fig. 3. Comparison of experimental wear depth measurements [4] (dashed line) with computed DEM simulation results (solid lines) in four different paths on the bend wall: A - without DRM, B - with DRM. 


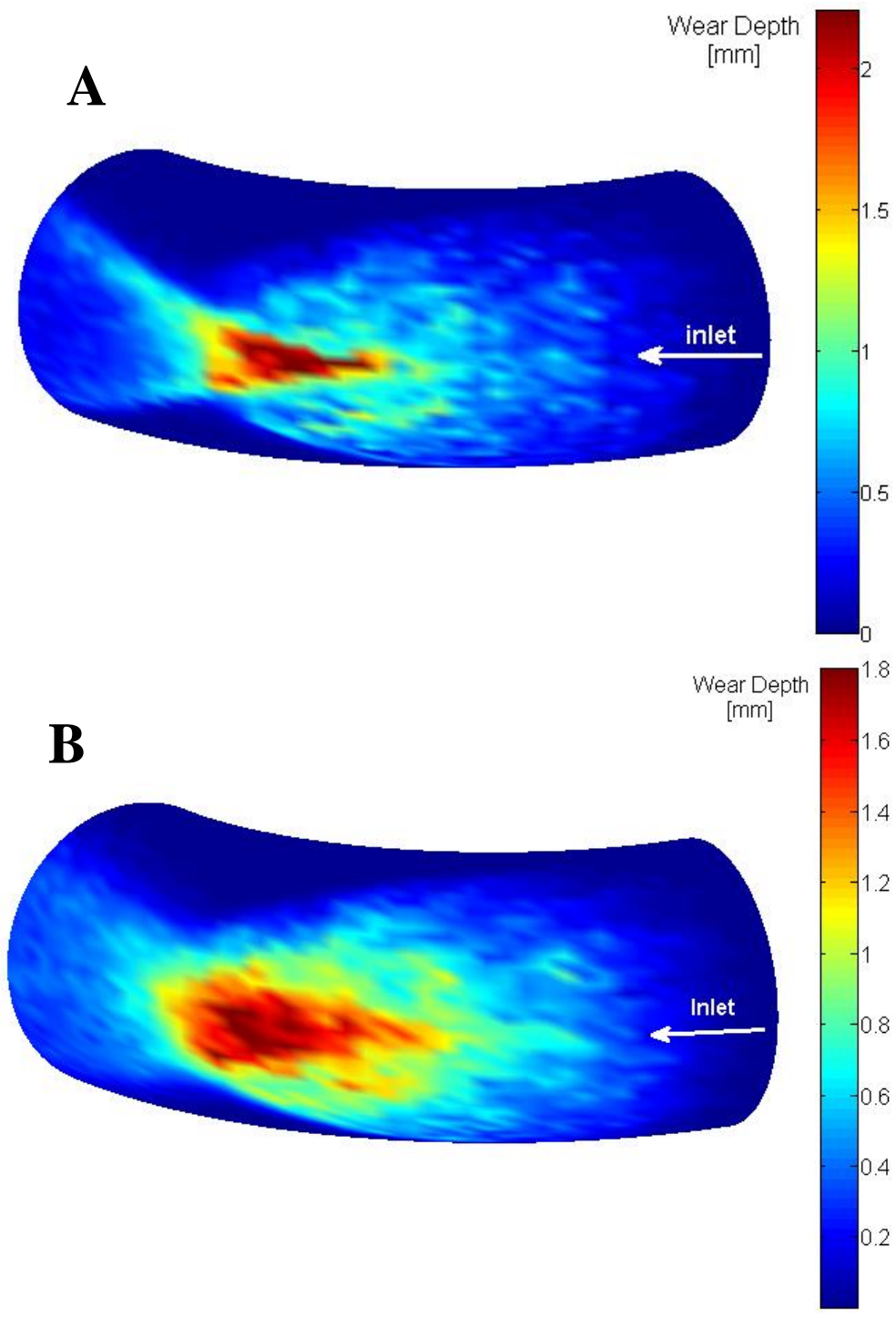

Fig. 4. Wear depth mapping of the DEM results: A - without DRM, B - with DRM.

\section{Application of ODEM: Pneumatic Conveying}

In the current study, the ODEM is implemented for a pneumatic conveying system of sand particles. This system consists of five straight sections and four bends, as shown in Fig. 5. In the following sections, this system is investigated in order to examine the applicability of ODEM. Both the particles dynamics and the erosion rate are compared against the 3D CFD-DEM simulations results in order to demonstrate the ability of ODEM to provide good assessments with low computational requirements. 


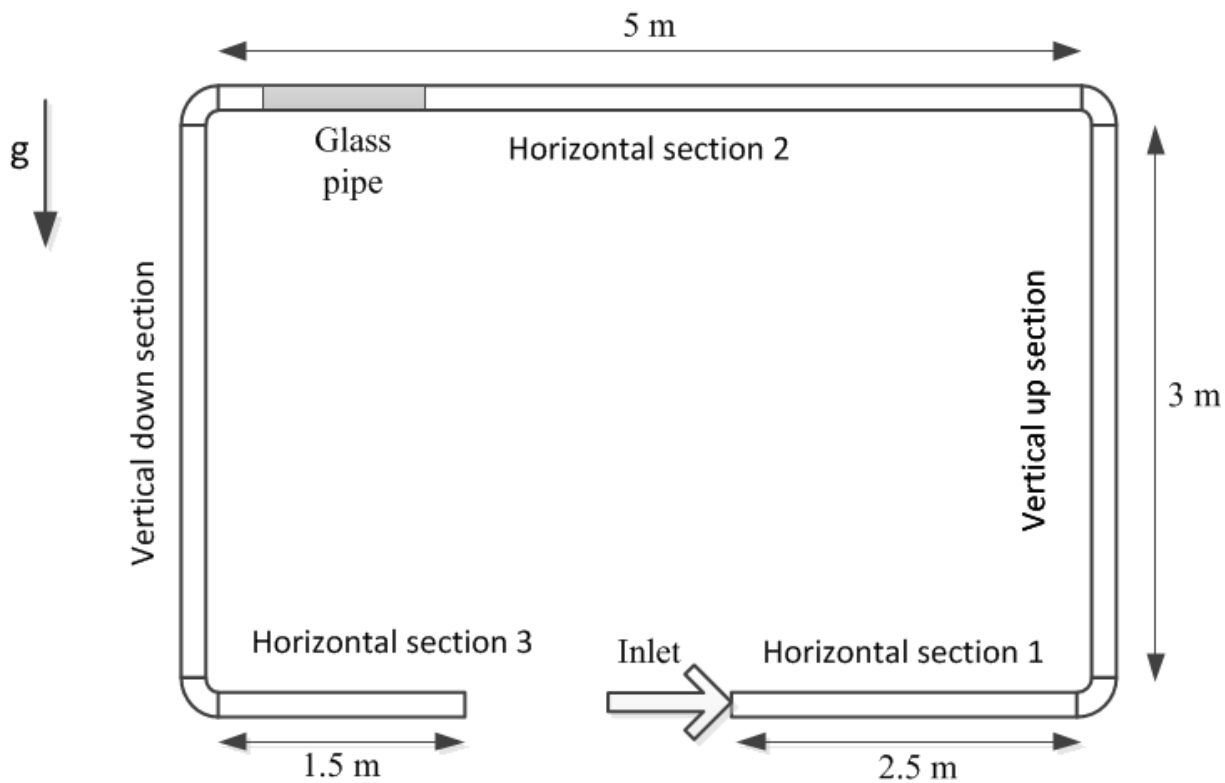

Fig. 5. Schematic illustration of the examined pneumatic conveying system; pipe diameter = 1 in.

\subsection{One-dimensional Two-phase Flow Validation}

The CFD-DEM simulation results serve as a basis for the validation of the one-dimensional flow model. The CFD-DEM simulation setup and the particle properties defined above in Error! Reference source not found. were used here as well; the simulated particle diameter was $250 \mu \mathrm{m}$ and the simulation boundary conditions were as follows: gas mass flux $=0.0584 \mathrm{~kg} / \mathrm{s}$, particle inlet velocity $=50 \mathrm{~m} / \mathrm{s}$, and mass flux $=0.034 \mathrm{~kg} / \mathrm{s}$. It is worth mentioning that the same setup was used here since the previous CFDDEM simulations were conducted to confirm the validity of the DEM method for erosion prediction with the implementation of the proposed models and properties.

The axial velocity of the particles was extracted from the 3D DEM results and is shown in Fig. 6. The results were analyzed after the transient simulation reached a steady particle mass flow rate, which was equal to that at the inlet. It is shown in the figure that the discrete particle velocity (blue dots) is quite scattered in the pipe cross section while the mean cross section velocity values (red dots) seem to follow a rather clear trend; from the inlet, the particles are accelerated in the straight section and then their velocity is damped significantly because of the collisions in the bend. This tendency is observed in every section that follows. It should be noted that the air is modeled as ideal gas; therefore, its density reduces when pressure drops along the line. This well-known phenomenon causes the gas velocity to increase along the line, and for this reason, the particle velocity does not reach a constant value. However, when the flow is fully developed the particles roughly following a constant velocity difference from the gas velocity, i.e. constant relative velocity. This is due to a linear increase of the gas velocity along the line, and when the 
particles follow the same linear trend it means that they reach their steady acceleration region. For the longest straight section (denoted as "Horizontal section 2" in Fig. 5), the particles appear to reach their relative terminal velocity since the velocity gradient of the particles is linear as can be observed in Fig. 6 (i.e., when the particles' relative velocity is constant, the acceleration is also constant).

From Fig. 6, it is well established that the one-dimensional two-phase results (black line) follow a similar trend as the DEM predictions. This ensures that the flow model predicts (according to the DEM) the time spent by the particles in each location with high accuracy, since it only depends on the particle phase velocity (Eq. 4).

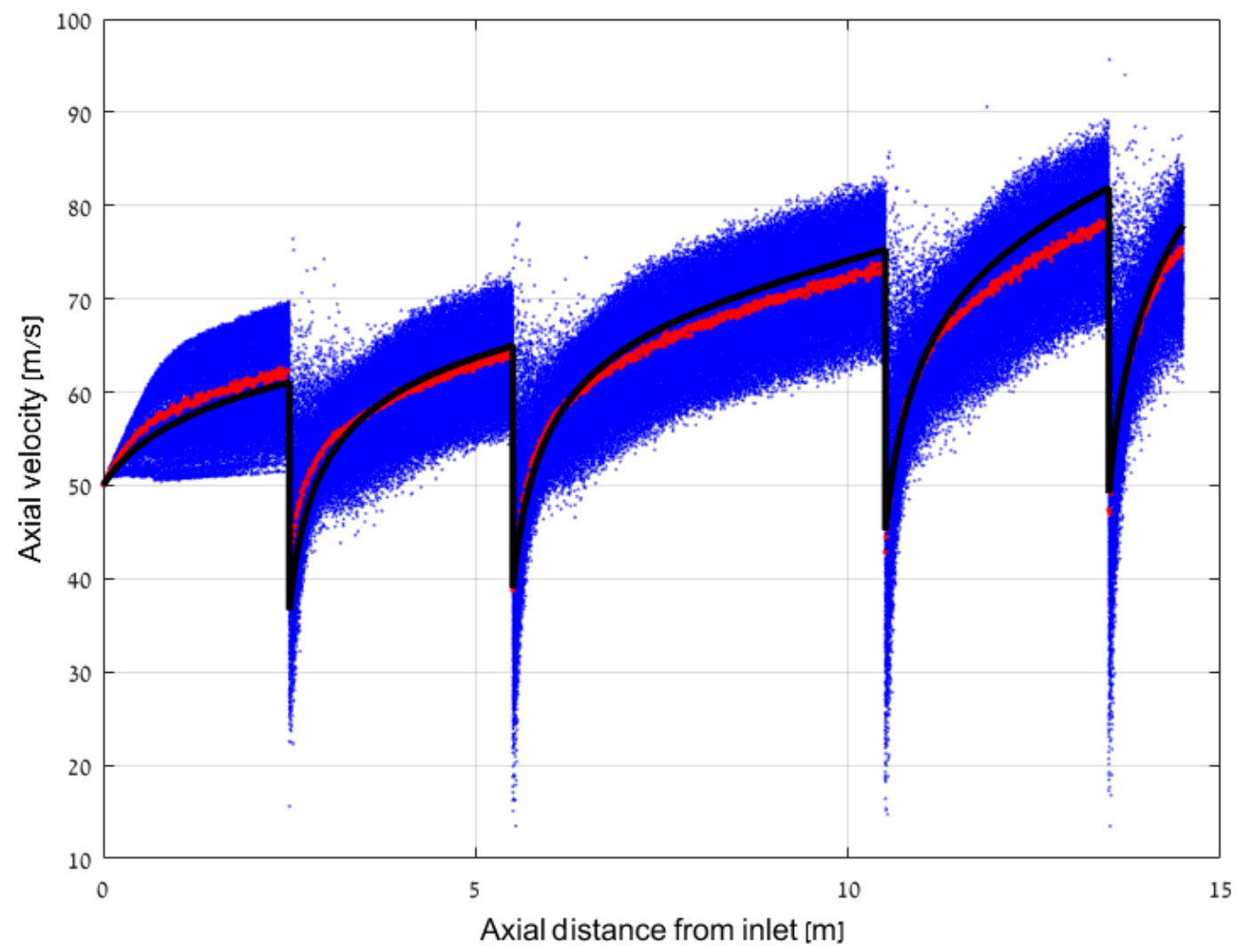

Fig. 6. Particle axial velocity results from CFD-DEM and the one-dimensional model. The scattered blue dots denote the discrete axial velocities and the red dots denote the mean velocity in the CFD-DEM simulation, while the black line represents the one-dimensional results.

\subsection{Collision Characteristic Functions}

The statistical distribution functions have to be developed in order to use the ODEM. In the current study, 3D CFD-DEM simulations are performed to develop the collision characteristic functions, although any other tool that can supply these functions can be applied in the ODEM. The same CFD-DEM simulations as those mentioned above used for the purpose of data analysis for the collision characteristic functions. 
To develop the statistical functions, the system's governing parameters must be determined first. The governing parameters define how to conduct the data analysis in terms of parameters that consist of similar dynamics. For example, every section in the system (i.e., the first horizontal pipe, first bend, vertical up-stream, second bend, second horizontal pipe, third bend, vertical down-stream, and third horizontal pipe) was assumed to have different statistical distribution functions and only one distribution for each.

Our study consists of a case that shares the same particle properties (size, material), gas properties, and geometry. These listed parameters were kept constant in the present analysis although they do, of course, govern the collision dynamics. For the pneumatic system under consideration, the governing parameters were assumed to be only the pipe geometry, as all the other parameters are constants.

The straight pipe sections in the examined pneumatic conveying system are connected by small-radius bends $(\mathrm{R} / \mathrm{D}=1)$. Therefore, in the undeveloped region after each bend, the 3D impact characteristics gradually change until the particles reach their stable state. Because the current study aims to develop an erosion prediction tool, the developed region is determined by the place at which the erosion rate calculated from the DEM data is constant. Our data show that the constant erosion rate region is obtained $\sim 60 \mathrm{D}(1.5 \mathrm{~m})$ after the bend for the straight pipe sections. This determination is based on the erosion rate results in straight pipes as shown in Fig. 13, while this subject will be further discussed in the results and disscussion section (5.3).

The analysis is thus divided into two parts: straight developed sections and bends. In order to obtain the collision characteristic functions in the undeveloped region, a blending function is proposed:

$$
f_{u d}=\tanh (x \pi) f_{b}+(1-\tanh (x \pi)) f_{d}
$$

where $f$ is any impact characteristic function, the subscript $u d$ denotes the undeveloped region, $d$ denotes the developed region, and $b$ denotes the bend function. Further, $x=x / x_{0}$ is defined as the nondimensional blending length, where $x$ is the distance from the bend outlet and $x_{0}$ is the blending length, which is assumed to be $1.5 \mathrm{~m}$. The logistic cumulative distribution function adopted for describing the different characteristic functions is given by

$$
s_{i}=\frac{1}{1+e^{\frac{f_{i}-\mu_{i}}{\lambda_{i}}}}
$$

where the subscript $i$ denotes a specific distribution function, such as collision frequency, collision velocity magnitude, impact angle with the wall surface, circumference impact angle, or bend angle. 
Further, $s_{i}$ is a random probability, $f_{i}$ is the distributed property, $\mu$ is the median, and $\lambda$ is the scale factor of the distribution.

The CFD-DEM collision data were collected after steady particle mass flux was reached at the outlet. The simulation continued to run for a period of $0.1 \mathrm{~s}$ to collect sufficient statistical collision data. Each collision of the discrete particles in the 3D domain was recorded during the simulation and saved to a file. Subsequently, the files were analyzed with respect to the collision characteristics governing parameters. The geometry dependence of the collision distribution functions were obtained by dividing the analysis to straight sections or the bends as follows.

\subsubsection{Straight Pipe Sections}

The impact velocity magnitude is defined as the magnitude of the particle's velocity in the first time step where there is a displacement between the particle and the wall, i.e., the velocity just before it collides with the pipe wall. The impact velocity magnitude distribution is given for all the straight sections shown in Fig. 7. As the particles are conveyed forward in the pipe sections, their impact velocity increases significantly; this can be deduced by simply examining their axial velocity in Fig. 6. However, the first horizontal section is excluded from this determination. It can be seen that the impact velocity of the particles in this section is distributed in a very narrow range. Furthermore, the impact angle of the first horizontal pipe section (Fig. 8) is significantly lower than that of the other sections, and it is distributed in a very small range. The divergence of the results of the first pipe section from those of the other sections is attributed to the inlet injection method in our DEM code. At the inlet, the particles are injected from a cartridge perpendicular to the inlet surface, and therefore, they are constructed in a random manner. After the first bend, the collisions within it cause mixture, and therefore, the initial arrangements are destroyed.

It can be seen from Fig. 7 that each section has a different distribution, and therefore, each one was fitted separately to provide its own distribution. The best fit for the velocity magnitude was obtained by applying the logistic distribution function to the cumulative CFD-DEM results. All the distribution fitting parameters with regard to every section are detailed in Table 2.

In contrast to the velocity distribution results, which shows differences, the impact angle (designated as $\alpha$ ) results share a similar distribution between the sections (excluding the first), as can be seen in Fig. 8 . Therefore, in order to simplify the model and reduce the number of independent statistical functions, it can be assumed that their mean distribution will provide a good assessment for this parameter in straight pipes. It should be noted that only the absolute value of this cumulative distribution should be considered for erosion calculation because the impact angle is always taken to be between $0^{\circ}$ and $90^{\circ}$. The impact angle results were duplicated with respect to $0^{\circ}$ as negative values to simplify the fitting procedure. The resulting logistic fitting has a median impact angle of zero and $\lambda_{\alpha}=4.315$. The joint impact angle 
distribution also provides an interesting conclusion; in fact, the impact angle in dilute pneumatic conveying in straight pipes (for the examined case) is nearly independent of the pipe orientation.

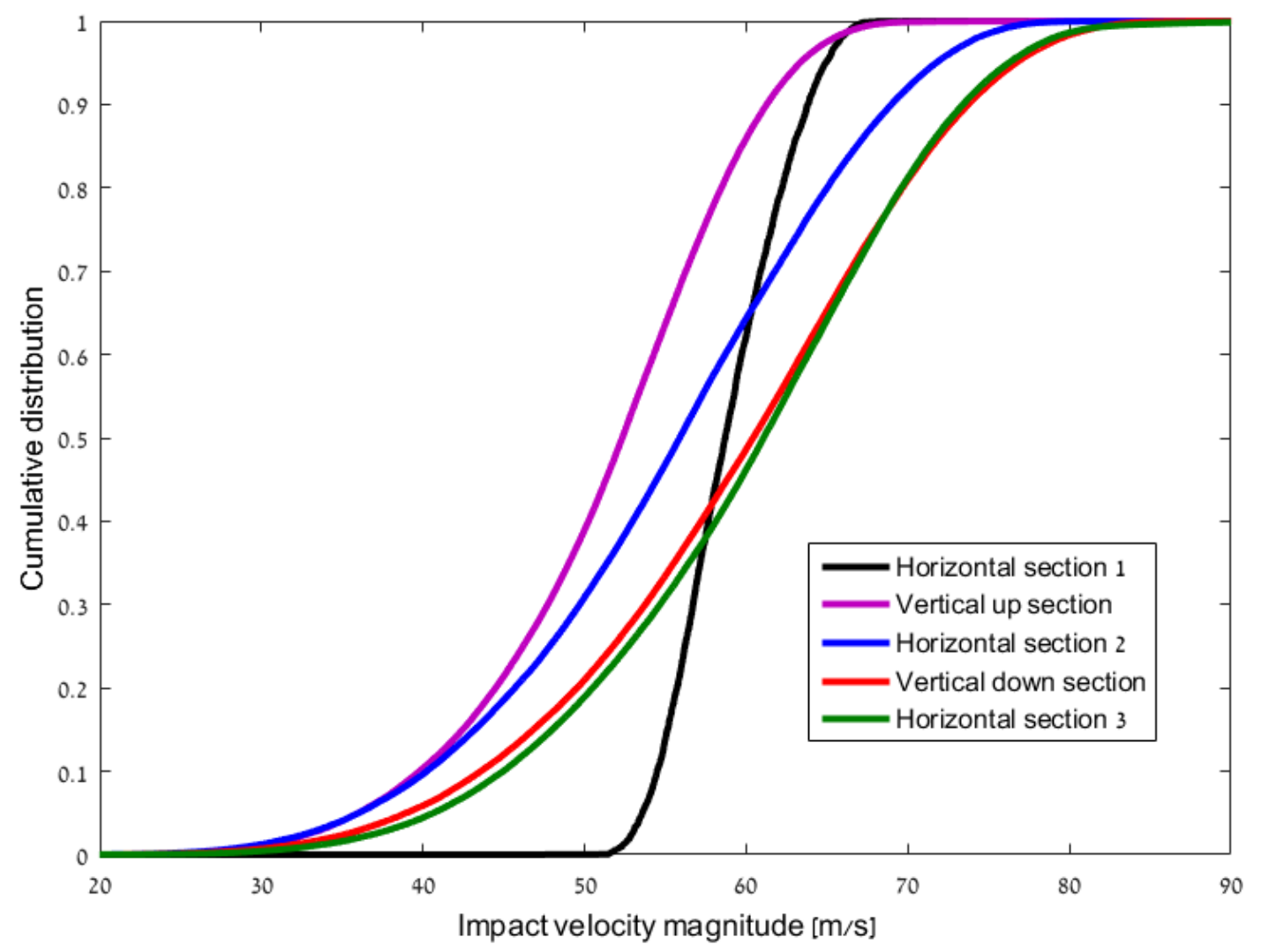

Fig. 7. Velocity impact magnitude distribution for all straight pipes. 


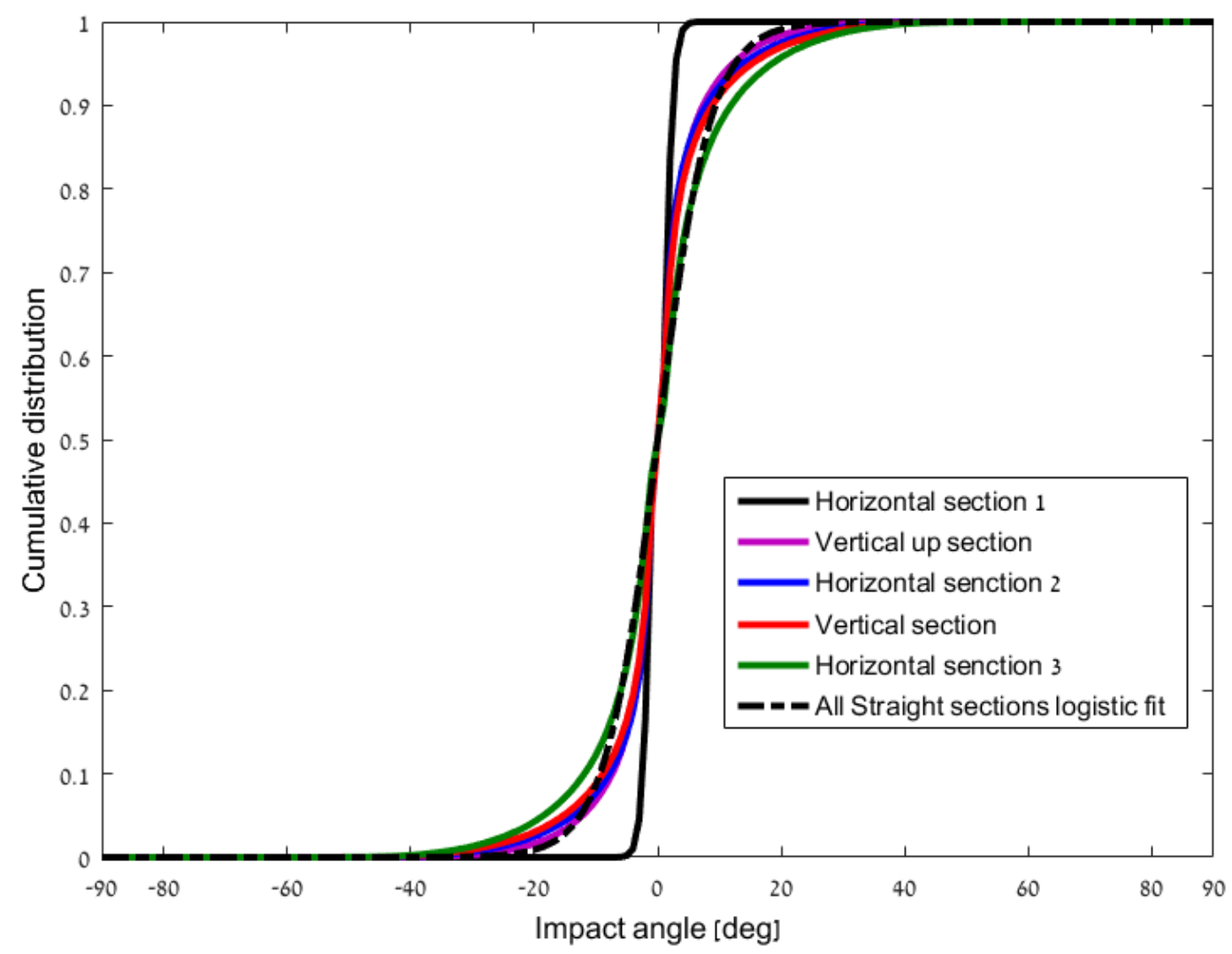

Fig. 8. Impact angle distribution for all straight pipes.

Remarkably, the circumference angle of the particle impact also seems to be independent of the pipe orientation, and it follows a nearly completely uniform trend (i.e., completely random distribution), as shown in Fig. 9. Therefore, the gravitational effects in this case are negligible and this parameter can be described by a uniform distribution.

The last examined parameter is the collision frequency, which was analyzed using the same procedure as that presented in our previous study [10] and represented by its median $\mu$ and scale parameter $\lambda$ (Table 2). 


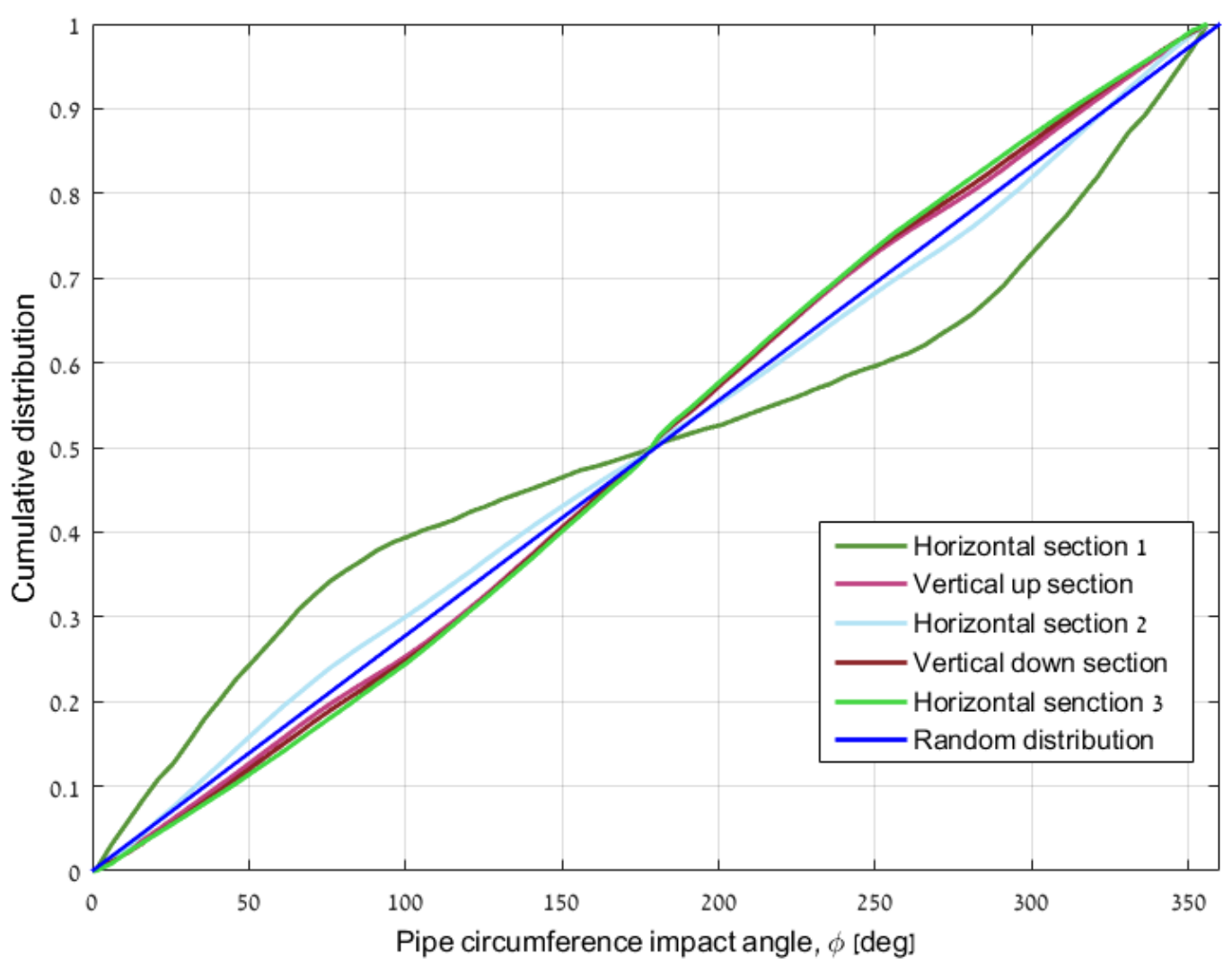

Fig. 9. Pipe circumference distribution for all straight pipes.

Table 2 Summary of the collision characteristic fitting parameters

\begin{tabular}{ccccc}
\hline & \multicolumn{2}{c}{ Collision frequency [Hz] } & \multicolumn{2}{c}{ Velocity magnitude $[\mathrm{m} / \mathrm{s}]$} \\
\hline & $\mu$ & $\lambda$ & $\mu$ & $\lambda$ \\
Horizontal section 1 & 4.125 & 2.03 & 58.83 & 2.058 \\
Vertical up section & 51.5 & 6.965 & 51.81 & 4.715 \\
Horizontal section 2 & 41.2 & 4.689 & 55.54 & 6.307 \\
Vertical down section & 75 & 10.61 & 59.9 & 6.653 \\
Horizontal section 2 & 82.5 & 5.069 & 60.5 & 6.317 \\
\hline
\end{tabular}

\subsubsection{Bends}

The distributions of the impact velocity magnitude inside the bends are given in Fig. 10A. As expected, the impact velocity inside the bends increases as the particles travel farther through the conveying system. The differences between the bends are considerable, and therefore, for each bend, a specific distribution function is fitted and described in Table 3. Nonetheless, when considering a non-dimensional 
representation of the velocity, i.e., the velocity magnitude divided by the median velocity, the resulting velocity distributions of the bends collapse into a single curve as shown in Fig. 10B. In the nondimensional form, the distribution results were fitted to a logistic distribution function, where its median value was 1 and the resulting scale factor of the distribution was 0.125 . This result means that there is no need to calculate the scale factors for each bend. However, the median of the velocity distribution must be estimated in order to reproduce the curves shown in Fig. 10A. Apparently, the predicted axial particle velocity at the bend inlet (from the one-dimensional flow model) correlates well with the median impact velocity. It was found that the velocities ratios were $0.82,0.83,0.84$, and 0.84 at the first, second, third, and fourth bends, respectively. Therefore, a constant value of 0.84 was used as the ratio between the median impact velocity and the bend inlet velocity.

When examining the velocity distribution results for straight pipes in their non-dimensional form, we also observe considerable similarity between the different sections, apart from the first horizontal and the vertical upstream pipes. In these cases, the ratio of the median to the mean axial velocity of the particles in each section (from the one-dimensional model) is $0.98,0.83,0.78,0.78$, and 0.79 with respect to the straight sections of the conveying system from the inlet and onward.

Remarkably, the non-dimensional form in the straight sections even correlates well with the nondimensional form that was obtained for the bends, as shown in Fig. 10B. Therefore, owing to the strong relationship between the different sections, it is suggested that this non-dimensional form could be used for a wide range of applications for estimating the distribution of the impact velocity of particles in dilute pneumatic conveyance. However, it should be noted that further investigation of this phenomenon is required to understand the range of applicability of these relations.

Table 3 Collision velocity magnitude distribution fitting parameters for logistic function

\begin{tabular}{ccc}
\hline & \multicolumn{2}{c}{ Velocity magnitude $[\mathrm{m} / \mathrm{s}]$} \\
\hline & $\mu$ & $\lambda$ \\
Bend 1 & 51.02 & 6.77 \\
Bend 2 & 53.56 & 7.09 \\
Bend 3 & 61.41 & 8.14 \\
Bend 4 & 65.73 & 9.19 \\
\hline
\end{tabular}

It can be deduced that the impact velocity in the bends and in the straight pipes are comparable, but it should be noted that in order to calculate the normal impact velocity, the velocity magnitude should be multiplied by the sine of the impact angle. Examining the impact angle distribution inside bends in Fig. 11A against the results of the straight section in Fig. 8 clarifies that, obviously, the normal velocity is 
significantly higher inside the bends (the median impact angle inside the bends is around $16^{\circ}$, compared to the relatively small median angle of $5^{\circ}$ in the straight pipes). Furthermore, Fig. 11A shows that inside bends, a similar profile describes the impact angle, regardless of the differences in the velocity magnitude. Therefore, the average of the impact angle distributions can describe one distribution function that represents all the bend impact angles, as shown in Fig. 11A.

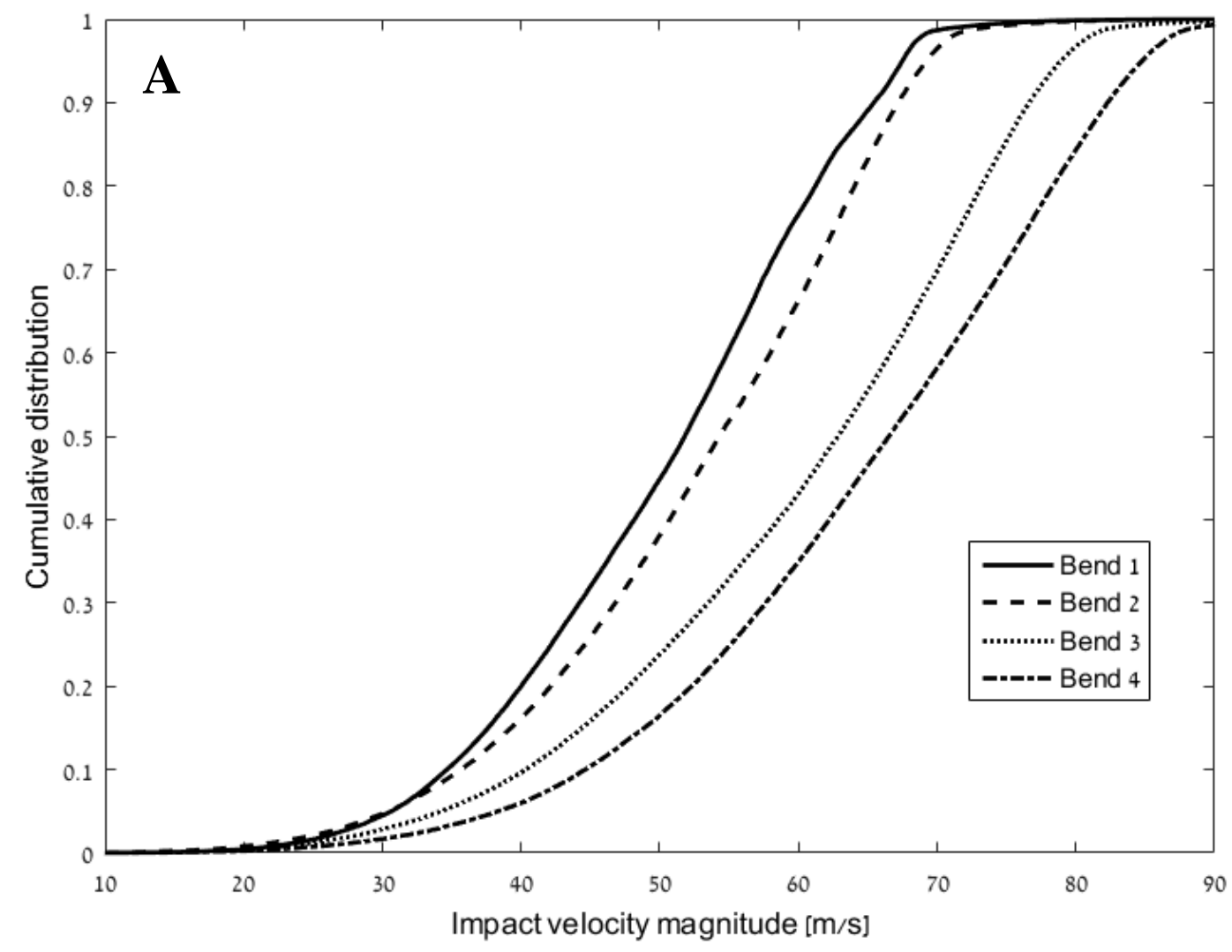




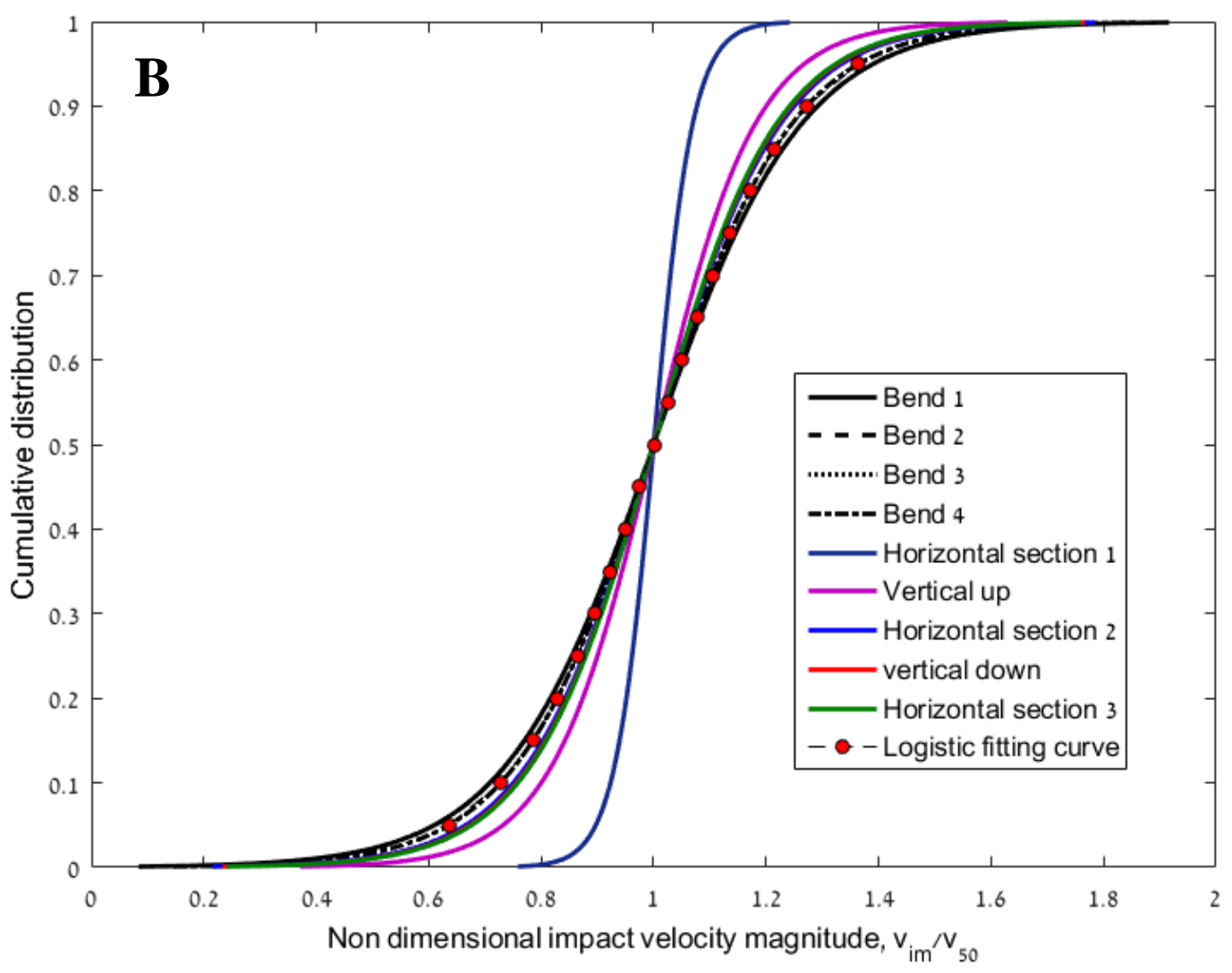

Fig. 10. Velocity impact magnitude distribution: A - all bends, B - non-dimensional representation of the velocity distribution for both straight pipes and bends.

The collision characteristics inside the bend also define the impact location along the bend, whereas the ODEM collision time defines the straight pipe impact location. This is provided by analyzing the distribution of the collisions along the bend angle, as shown in Fig. 11B. It is found that this parameter is also independent of the bend number, similar to the impact angle, because the results share a relatively similar tendency. For this reason, the bend angle distribution can be described qualitatively by one logistic distribution function $\left(\mu_{\theta}=53\right.$ and $\left.\lambda_{\theta}=13.77\right)$ that is given by the mean of all four bend values. 

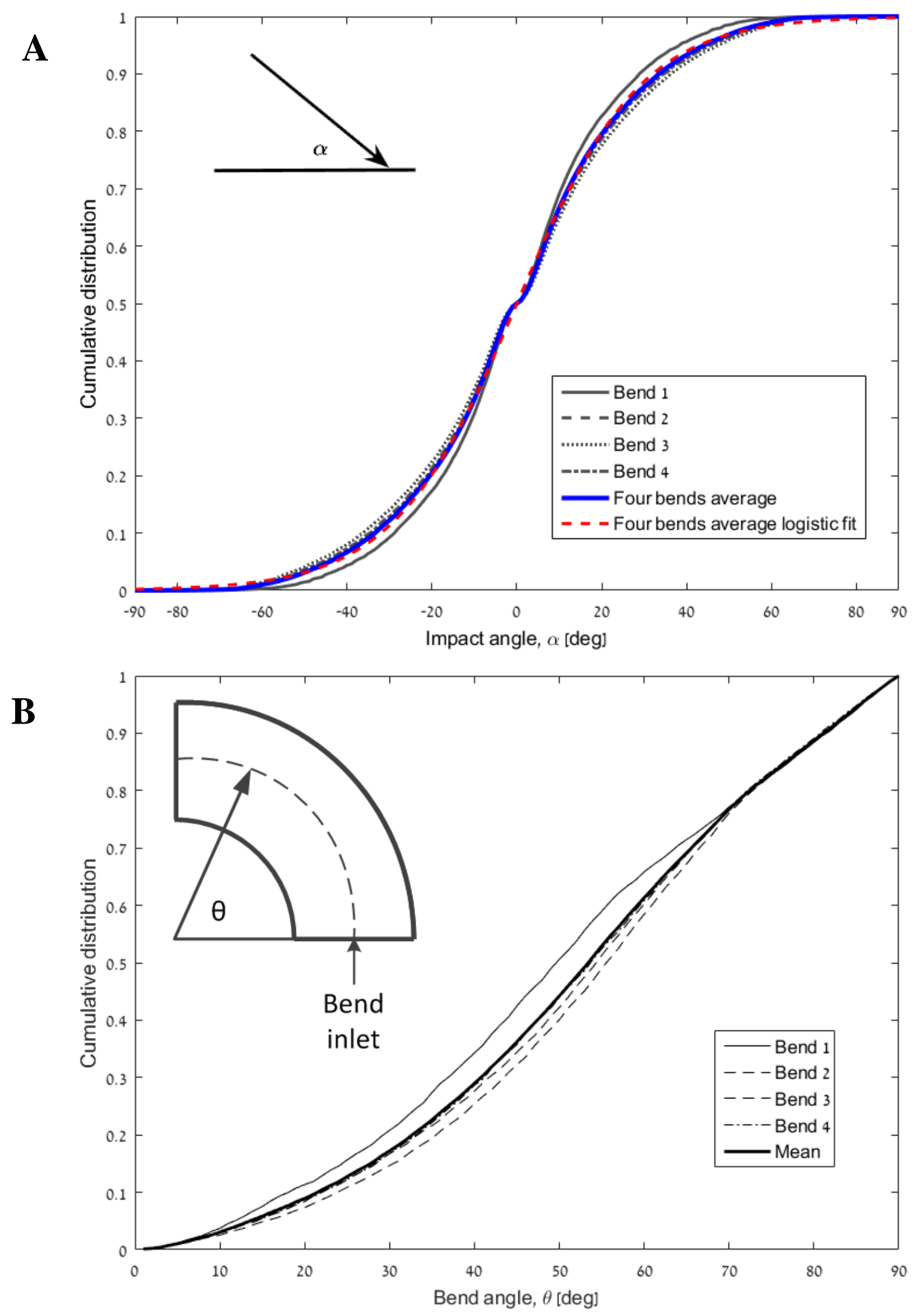


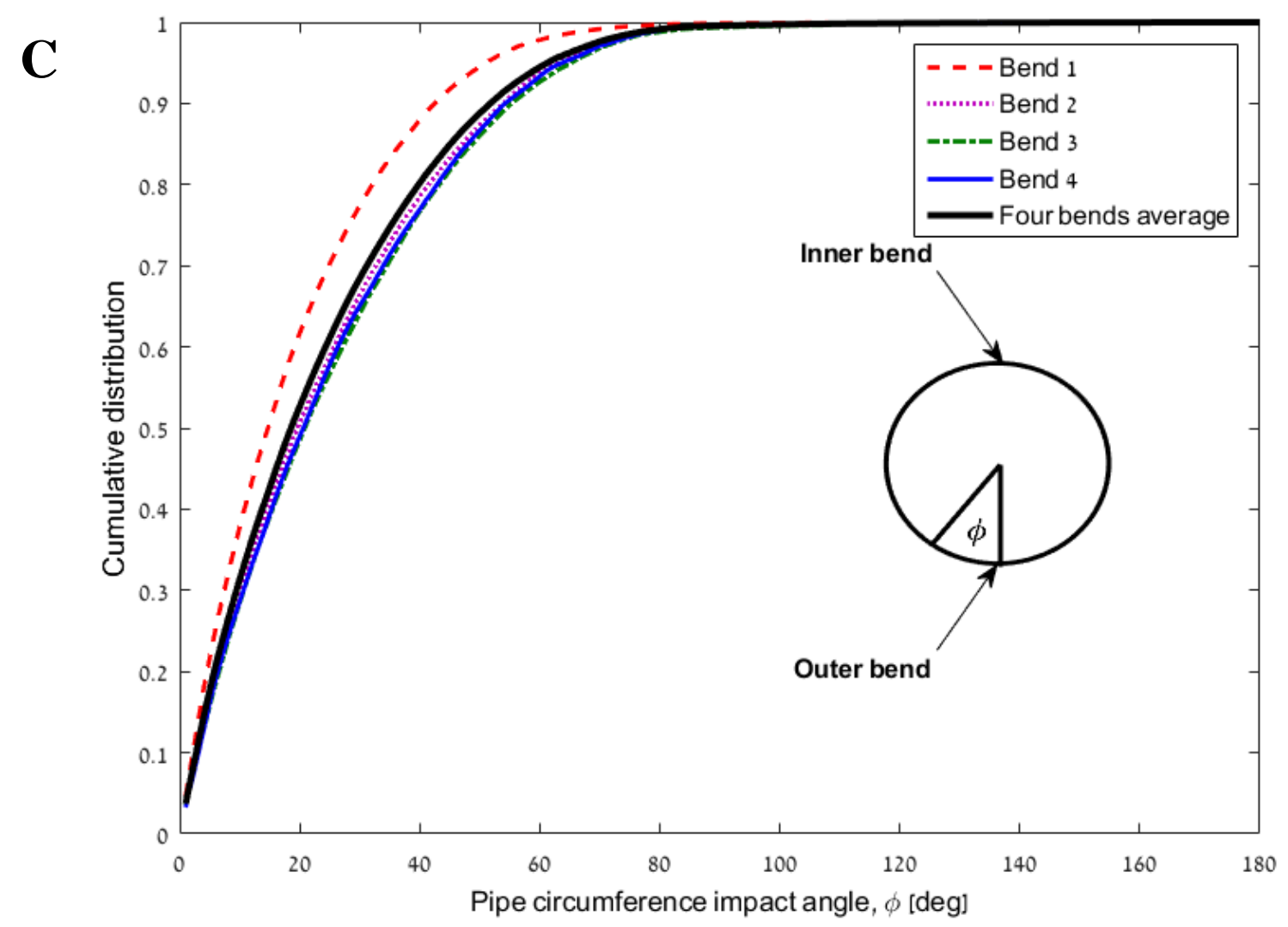

Fig. 11. Cumulative distribution of the impact inside bends. A - Impact angle, B - bend impact angle ( $\theta)$, C pipe circumference impact angle.

The last parameter that characterizes the collision dynamics inside the bends is the circumference impact angle that determines the circumference pipe location of the collision. Fig. 11C shows the distribution of the circumference impact angle which correspond to the mean of all bend angles; once again, the mean distribution provides a good estimation for all the bends in the system. However, unlike other parameters, the circumference impact angle is found to be dependent on another parameter: the bend angle.

The variance of the circumference impact angle differs significantly at different bend angles, but the median circumference angle is constant owing to symmetry. Therefore, $\lambda$ was examined in terms of the bend angle. The scale factor $\lambda_{\phi}$ was found by fitting the CFD-DEM data to the logistic distribution function at every bend angle with a spread of $1^{\circ}$ along the bend, as shown in Fig. 12. In order to determine the relation between the circumference impact angle and the bend angle by a simple function, these results were fitted to a polynomial of degree 6 (with a constraint that the derivative at $\theta=0^{\circ}$ is zero):

$$
\lambda_{\phi}=p_{0}+p_{1} \theta+p_{2} \theta^{2}+p_{3} \theta^{3}+p_{4} \theta^{4}+p_{5} \theta^{5}+p_{6} \theta^{6}
$$

where the constants are presented in ascending order of their subscripts as follows: 21.58, 0, -0.02043, $0.001994,-6.731 \times 10^{-5}, 8.87 \times 10^{-7},-3.958 \times 10^{-9}$. It should be noted that the given distribution in Fig. $11 \mathrm{C}$ is 
therefore an inaccurate estimation to be used for all bend angles, but it is provided as a mean to quantitatively exemplify the circumference impact angle distribution.

From the above analyses, it was deduced that the particle collision dynamics are similar for all the bends along the conveying line even though each bend has a different orientation. This indicates that the flow pattern of the particles is similar and the gravitational effects are negligible.

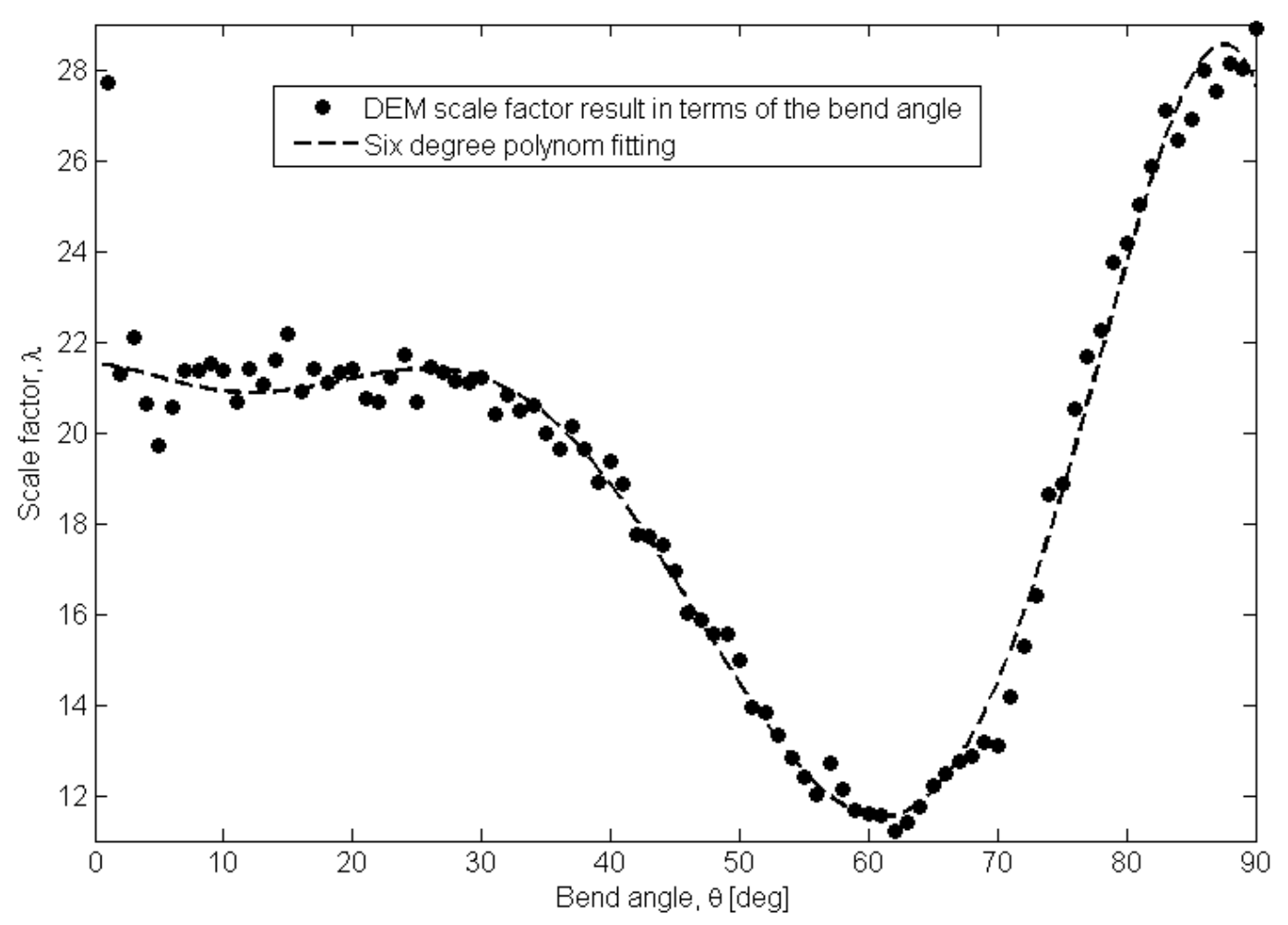

Fig. 12. Scale factor in terms of the bend angle.

In the ODEM, the erosion rate at a specific point in the bend depends on the number of collisions per particle with the bend walls and four statistical functions: the bend angle, circumference angle, impact angle, and impact velocity magnitude. The implicit assumption in this approach is that the parameters are independent of one another, except for the circumference angle, which is calculated in terms of the bend angle. The collapse of the collision characteristics into those parameters while providing a single statistical distribution for every parameter highly simplifies the process of erosion prediction, i.e., with this approach, every parameter could be measured or evaluated separately regardless of the other parameters, and then, those measurements could be implemented for a rather simple simulation in order to obtain the erosion rate. 
Because the particle dynamics inside the bends is not calculated in the ODEM, the collision frequency inside bends is irrelevant. Therefore, the number of collisions per bend was estimated from the DEM data and adopted in the ODEM. Three collisions per particle per bend were typical of the bends, and only a negligible deviation was observed.

A problem arises when trying to evaluate the frequency distribution in the undeveloped straight sections using the blending function. This is because the collision frequency for bends (typically three impacts per particle) is not suitable for the straight pipe just after the bend, and therefore, it cannot be used in the blending functions. In order to deal with this problem, a length of $10 \mathrm{~cm}$ after the bend was considered to evaluate the frequency with regard to the bend in the blending function. It was deduced that its median frequency is $1300 \mathrm{~Hz}$ and the scale factor of the logistic fitting is 200.6.

\subsection{ODEM Results and Discussion}

Similar to the DEM simulation, during the ODEM simulation, the collision places and the impact characteristics of each collision (velocity magnitude, impact angle, etc.) are saved for post processing. In order to provide erosion mapping of the pipe walls, a numerical mesh was used to project every collision location from the DEM/ODEM data to the corresponding place in the geometry. The erosion depth was thus calculated by computing the volume of material that was removed using Eq. (5) and dividing it by the area of the face to which the collision was projected. However, the erosion rate is the erosion depth divided by the particle mass conveyed through the system (in the steady state, the conveyed mass is simply the integration of the mass flux in terms of the simulation time).

The erosion rate of straight sections is shown in Fig. 13 in terms of the axial distance from the conveying system's inlet. In the figure, the results correspond to the outer part of the pipe wall $\left(-10^{\circ 0}<\phi<10^{\circ}\right)$, and the bend erosion rate is not displayed in this plot. The erosion rate in the first horizontal section is negligible because the particles rarely impact the wall as discussed earlier in Section 5.1. After the bends, Fig. 13 shows that the blending function provides a very good match with the DEM erosion rate in the undeveloped straight sections. For straight developed sections, the erosion rate assessments of the ODEM are also comparable to the calculated DEM erosion rate, as shown clearly in Fig. 13. 

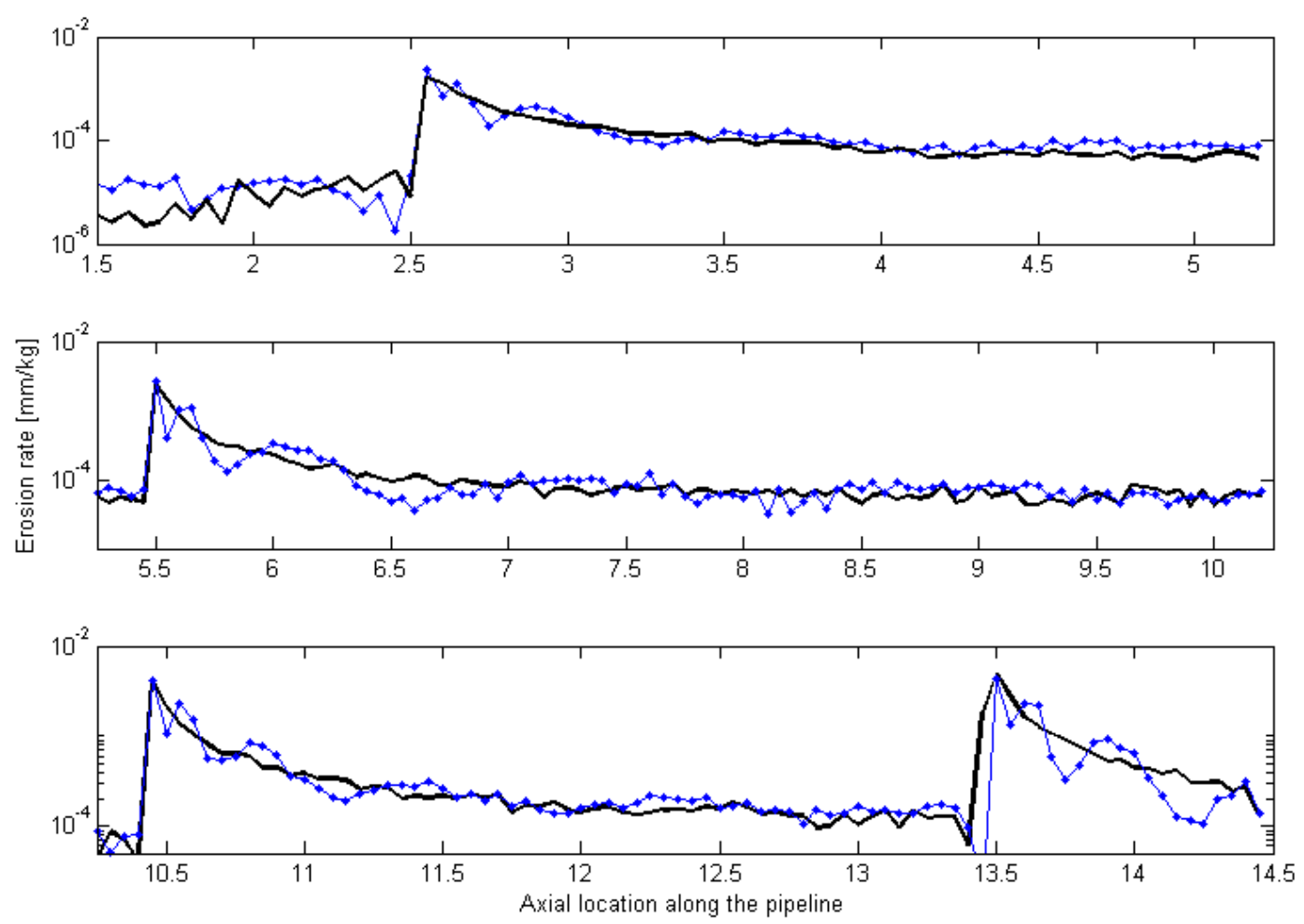

Fig. 13. Erosion rate of straight sections: ODEM - blue dotted line, CFD-DEM - black line.

The erosion map of the entire system can be provided using either the ODEM or the DEM. However, since the straight pipe is not eroded in one preferred circumference direction, the $3 \mathrm{D}$ erosion mapping is much more beneficial for bends. Bends are well known as the most vulnerable parts in a pneumatic conveying system, but the particle dynamics is much more complicated to predict. For this reason, erosion prediction in bends is one of the most critical and challenging tasks.

The ODEM and the DEM erosion rate results are mapped and displayed for the second bend in Fig. 14. It can be seen in Fig. 14B that the erosion distribution near the bend exit at the circumference of the pipe, where the particle collisions with the bend wall are secondary (or more), is scattered owing to the effect of the DRM. The erosion rate is obviously much higher in the outer bend wall, which is prominent for both the ODEM results (Fig. 14A) and the DEM results (Fig. 14B). Furthermore, the ODEM results share a very similar erosion map with the DEM results. It can be concluded from the figures that the statistical analysis developed for the ODEM establishes an excellent tool to predict the 3D erosion rate in the bend compared to the original DEM results. 


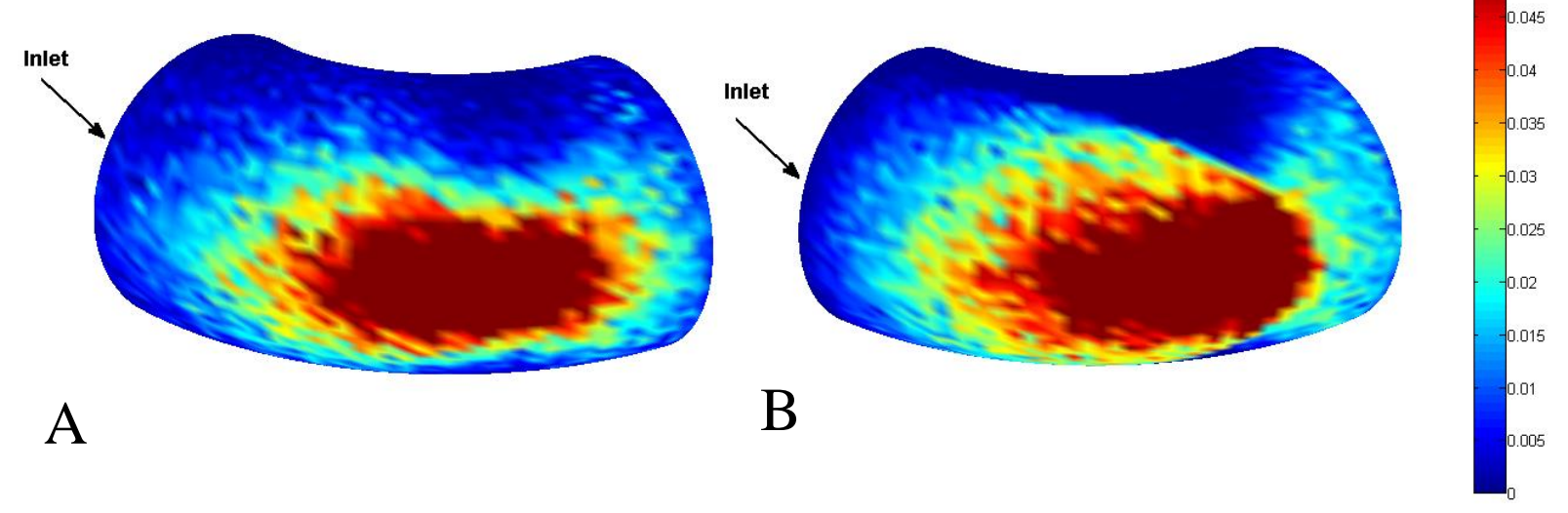

Fig. 14. Erosion rate contour of the second bend in $\mathrm{mm} / \mathrm{kg}$ : A - ODEM, B - CFD-DEM.

The erosion rate results at the outer bend line $\left(-10^{\circ}<\phi<10^{\circ}\right)$ for the four bends in the conveying system are plotted in Fig. 15. It can be seen from the plot that the results of the ODEM for the first bend do not follow the same trend as the DEM results. The reason for this deviation is that the same collision characteristic functions were used for all the bends, although the impact characteristic of the first bend differed from the rest, as shown in Section 5.2.2. Of course, this could be easily overcome by fitting the collision characteristics to the data of the first bend, but it would degrade the ODEM general approach.

For the second, third, and fourth bends, Fig. 15 shows that the erosion rate calculated using the ODEM is strongly correlated to the prediction of the 3D CFD-DEM. The maximum erosion rate is obtained at a bend angle of $56^{\circ}-60^{\circ}$ for all bends, while in the ODEM, it only depends on the statistical distribution of the bend angle; median bend angle distribution (Fig. 11B) naturally agrees with this value. However, the maximum value of the erosion rate increases with the bend number in the same manner that the velocity magnitude changes (see Fig. 10A). Therefore, the collision characteristics are the most important aspect while predicting erosion with the ODEM and they share significant insight about the erosion of the system even without running the simulation.

The collision characteristics functions that were used in the ODEM were developed from DEM data while assuming that they are statistically independent. Furthermore, these functions were not calibrated to increase accuracy. Therefore, this methodology is based on very simplifying assumptions, but yet it is capable of providing trustworthy erosion rate predictions. The ODEM is evident by this comparison as a fine tool to predict erosion with an accuracy which is comparable to the conservative DEM. 


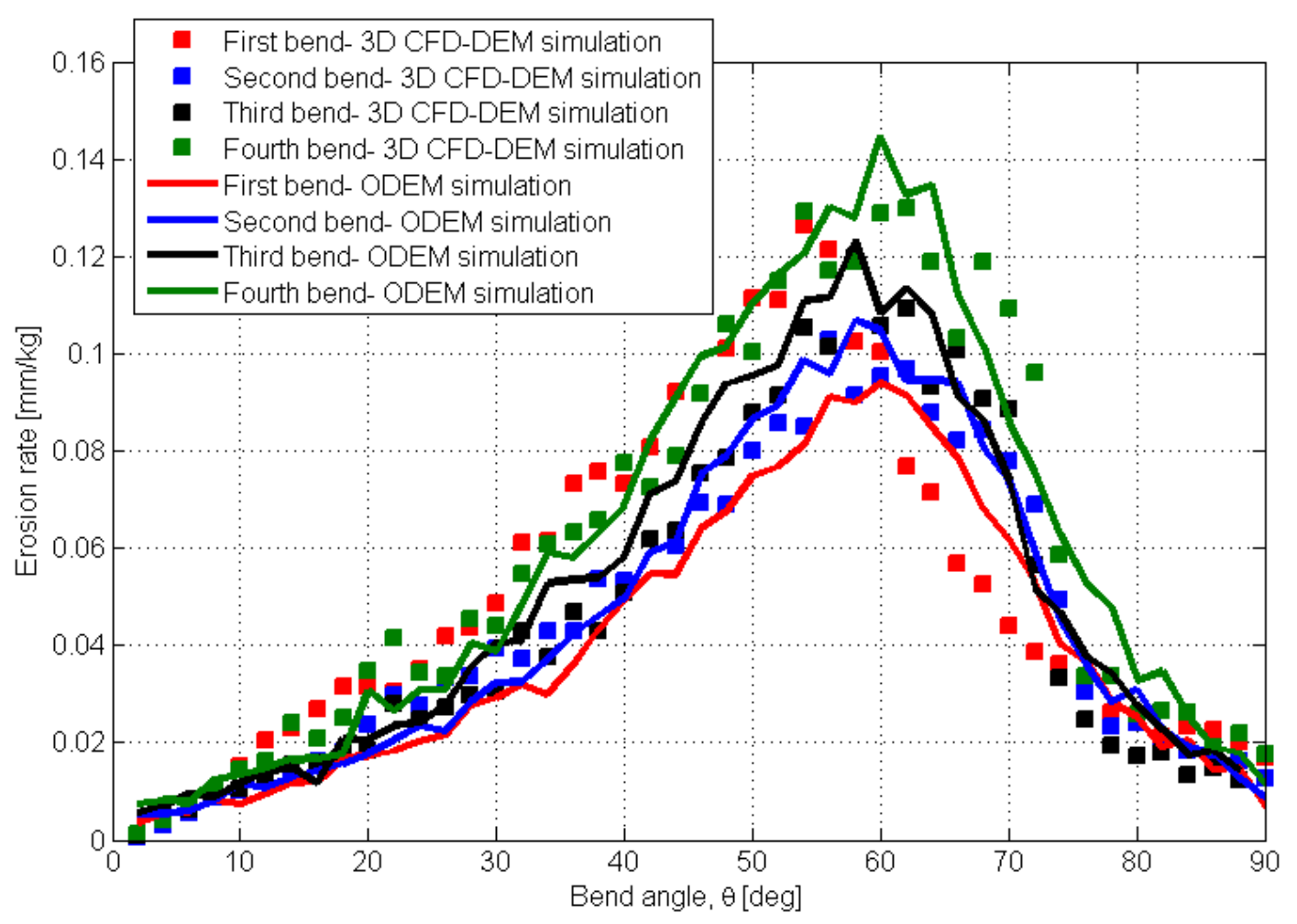

Fig. 15. Erosion rate results in terms of the bend angle: ODEM (lines) and CFD-DEM (dots).

One of the most important incentives for developing the ODEM is to reduce the computational cost compared to the expensive 3D CFD-DEM simulations. In order to compare the two, the ODEM computation time and the physical simulation time should be clarified with respect to the CFD-DEM.

The ODEM tracks a sample of particles, considering steady-state flow. Nevertheless, the tracking procedure searches for particle collisions with regard to the collision time inside an element. The chosen size of the tracked mass and the calculated particle volume fraction determine the size of the element (Eq. 3 ), and therefore, they determine the residence time of the tracked mass inside the element. However, in practice, the domain contains many more particles (as in the 3D DEM) than those within the tracked mass. During the residence time that the tracked mass is considered inside an element, other particles in the domain are moving as well, but they are not tracked by the ODEM. Thus, when simulating the ODEM, the period of time that should be considered with regard to the transient movement of all the particles in the domain is the traveling time inside one element (here, for demonstration purposes, we assume uniform particle velocity and volume fraction throughout the system).

The ODEM simulation was conducted with a tracked mass composed of 400 particles, and the boundary conditions were the same as those specified for the DEM simulations. The overall traveling time from the inlet to the outlet (i.e., one conveying round in the ODEM) of this mass was $\sim 0.4$ s. Equivalently, in one DEM round ( $0.4 \mathrm{~s}$ of simulation time), we tracked $\sim 380 \mathrm{~K}$ particles. Thus, the ratio of the number of 
tracked particles of the DEM to that of the ODEM was 950. Considering this ratio, the physical DEM time for tracking the same number of particles in one round of the ODEM is $0.4 / 950=4.5 \times 10^{-4} \mathrm{~s}$. The computational time of each round of the ODEM was $115 \mathrm{~s}$, while simulating $4.5 \times 10^{-4} \mathrm{~s}$ with the DEM took $1.73 \mathrm{~h}$. Thus, the computational time ratio was $1 / 54$ despite the fact that the DEM was performed using a 48-core CPU (Intel ${ }^{\circledR}$ Xeon $^{\mathrm{TM}}$ E5-2690 [2.6 GHz], and 256 GB RAM on LINUX CentOS 5.7) whereas the ODEM was run on a MATLAB ${ }^{\mathrm{TM}}$ platform using a serial CPU (Intel ${ }^{\circledR}$ Core $^{\mathrm{TM}}$ i7-4930K, with 8 GB RAM).

Sufficient statistical detail was obtained by conducting 80 conveying cycles with the ODEM, which took $2.5 \mathrm{~h}$. On the other hand, the transient CFD-DEM simulation which run for a period of $0.1 \mathrm{~s}$ (after steady mass flux was reached), which took 16 days.

\section{Conclusions}

In this paper, a new concept of the ODEM was presented, validated, and implemented for a pneumatic conveying system. The ODEM is a combination of a one-dimensional flow model and statistical distribution functions that describe the collision characteristics. CFD-DEM simulations served as a numerical tool for deriving the statistical functions of collision characteristics and as a basis for ODEM validation. The DEM code was modified in the current study to consider the effects of drag due to turbulence (random walk model), particle-wall rebound (the dimple rebound model), and particle shape effects on the drag force (in terms of sphericity). In order to validate our CFD-DEM simulation as a reliable tool for erosion prediction using the single-particle-impact erosion model, the experimental measurements of Solnordal et al. were compared with the CFD-DEM results and they were found to be in satisfactory agreement.

Both the ODEM and the CFD-DEM were implemented for the same pneumatic conveying system, and the ODEM predictions were validated against the CFD-DEM data. Both models showed similar results for the particle axial velocity, which implies that the dynamic predictions of the ODEM are valid. The CFD-DEM data were analyzed to obtain statistical functions that describe the collision characteristics required for the ODEM. The statistical functions derived for the straight pipe sections were collision frequency, circumference angle, impact angle, and impact velocity magnitude. Furthermore, the bend angle and the aforementioned functions were developed for bends. It was found that all the bends shared the same distribution of bend angle, impact angle, and circumference angle. The impact velocity magnitude and the collision frequency were the only parameters that were not similar for all bends and straight sections (mostly because of air expansion). Nonetheless, the impact velocity distribution divided by its median value leads to a non-dimensional form that collapses all the bend and straight section 
distributions into a single curve. This indicates that the non-dimensional form correlates a large range of parameters. Furthermore, the median impact velocity was found to correlate with the axial velocity, and therefore, it could be estimated explicitly from the one-dimensional flow model, regardless of the specific 3D simulation results or experimental measurements. Hence, the non-dimensional form is highly applicable. However, it should be noted that further investigation is required to clarify the range of its applicability.

The statistical functions describe each parameter independently of one another, except for the circumference angle, which was calculated in terms of the bend angle. This means that these parameters could be measured or computed regardless of the others (no statistical correlation between the functions is required), thereby facilitating fast and easy erosion prediction if those functions are known.

The erosion rate results of the ODEM and the DEM across the entire conveying line exhibited good agreement. This validates the ODEM as a tool for predicting the erosion rate in long industrial conveying lines, including bends and straight sections. This tool is much faster than a direct 3D CFD-DEM simulation (calculation time ratio of 1/54). Furthermore, the main advantage of the new model lies in its ability to upscale or perform parameter optimization and finally predict erosion in realistic conveying systems. However, the developed functions have to be generalized in terms of the material and wall properties in order to extend the applicability of the ODEM.

\section{REFERENCES}

[1] Y.I. Oka, K. Okamura, \& T. Yoshida, Practical estimation of erosion damage caused by solid particle impact: Part 1: Effects of impact parameters on a predictive equation, Wear 259(1) (2005) 95-101.

[2] K.R. Ahlert, Effects of particle impingement angle and surface wetting on solid particle erosion of AISI 1018 steel (Ph.D. Thesis), Department of Mechanical Engineering. The University of Tulsa, 1994.

[3] Y. Zhang, E.P. Reuterfors, B.S. McLaury, S.A. Shirazi, E.F. Rybicki, Comparison of computed and measured particle velocities and erosion in water and air flows, Wear 263 (2007) 330-338.

[4] C.B. Solnordal, C.Y. Wong, \& J. Boulanger, An experimental and numerical analysis of erosion caused by sand pneumatically conveyed through a standard pipe elbow, Wear 336 (2015) 43-57.

[5] R.E. Vieira, A. Mansouri, B.S. McLaury, \& S.A. Shirazi, Experimental and computational study of erosion in elbows due to sand particles in air flow, Powder Technol 288 (2016) 339-353.

[6] W. Peng \& x. Cao, Numerical simulation of solid particle erosion in pipe bends for liquid-solid flow, Powder Technol 294 (2016) 266-279.

[7] R. Zhang, H. Liu, \& C. Zhao. A probability model for solid particle erosion in a straight pipe, Wear 308(1) (2013) 1-9. 
[8] H. Liu, Z. Zhou, \& M. Liu, A probability model of predicting the sand erosion profile in elbows for gas flow, Wear 342 (2015) 377-390.

[9] W. Tabakoff \& A. Hamed, Aerodynamic effects on erosion in turbomachinery, CINCINNATI UNIV OHIO, 1997.

[10] A. Uzi, H. Kalman, \& A. Levy, A novel particle attrition model for conveying systems, Powder Technol 298 (2016) 30-41.

[11]Ben-Ami, Y., Uzi, A., \& Levy, A. Modelling the particles impingement angle to produce maximum erosion. Powder Technol, 301 (2016) 1032-1043.

[12] Bitter, J. G. A. A study of erosion phenomena part I. wear, 6(1) (1963) 5-21.

[13] Neilson, J. H., \& Gilchrist, A. Erosion by a stream of solid particles. Wear, 11(2) (1968) 111-122.

[14]Huang, C., Chiovelli, S., Minev, P., Luo, J., \& Nandakumar, K. A comprehensive phenomenological model for erosion of materials in jet flow. Powder Technology, 187(3) (2008) 273-279.

[15] Arabnejad, H., Mansouri, A., Shirazi, S. A., \& McLaury, B. S. Development of mechanistic erosion equation for solid particles. Wear, 332 (2015) 1044-1050.

[16] Finnie, I. Erosion of surfaces by solid particles. Wear, 3(2) (1960) 87-103.

[17] C.Y. Wong, C. Solnordal, A. Swallow, S. Wang, L. Graham, \& J. Wu, Predicting the material loss around a hole due to sand erosion, Wear 276 (2012) 1-15.

[18] T. Brosh, H. Kalman, \& A. Levy, Fragments spawning and interaction models for DEM breakage simulation, Granul Matter 13(6) (2011) 765-776.

[19] T. Brosh, H. Kalman, \& A. Levy, DEM simulation of particle attrition in dilute-phase pneumatic conveying, Granul Matter 13(2) (2011) 175-181

[20]A. Haider \& O. Levenspiel, Drag coefficient and terminal velocity of spherical and nonspherical particles, Powder Technol 58(1) (1989) 63-70.

[21] G.R. Desale, B.K. Gandhi, \& S.C. Jain, Effect of erodent properties on erosion wear of ductile type materials, Wear 261(7) (2006) 914-921.

[22] A.D. Gosman \& E. Loannides, Aspects of computer simulation of liquid-fueled combustors, J Energy 7(6) (1983) 482-490.

[23] Y. Tsuji, T. Tanaka, \& T. Ishida, Lagrangian numerical simulation of plug flow of cohesionless particles in a horizontal pipe, Powder Technol 71(3) (1992) 239-250.

[24] A.A. Cenna, N.W. Page, K.C. Williams, M.G. Jones, Wear mechanisms in dense phase pneumatic conveying of alumina, Wear 264(11-12) (2008), 905-913.

[25]M. Sommerfeld \& N. Huber, Experimental analysis and modelling of particle-wall collisions, Int J Multiphase Flow, 25(6) (1999) 1457-1489.

[26] Y.I. Oka, M. Matsumura, \& T. Kawabata, Relationship between surface hardness and erosion damage caused by solid particle impact, Wear 162 (1993) 688-695. 